\title{
Creating a Test-Validated Finite-Element Model of the X-56A Aircraft Structure
}

\author{
Chan-gi Pak* and Samson Truong \\ NASA Armstrong Flight Research Center, Edwards, California 93523-0273
}

DOI: $\underline{\text { 10.2514/1.C033043 }}$

\begin{abstract}
Small modeling errors in a finite-element model will eventually induce errors in the structural flexibility and mass, thus propagating into unpredictable errors in the unsteady aerodynamics and the control law design. One of the primary objectives of the X-56A Multi-Utility Technology Testbed aircraft is the flight demonstration of active flutter suppression and, therefore, in this study, the identification of the primary and secondary modes for the structural model tuning based on the flutter analysis of the $\mathrm{X}-56 \mathrm{~A}$ aircraft. The ground-vibration test-validated structural dynamic finite-element model of the $\mathrm{X}-56 \mathrm{~A}$ aircraft is created in this study. The structural dynamic finite-element model of the X-56A aircraft is improved using a model-tuning tool. In this study, two different weight configurations of the $X-56 \mathrm{~A}$ aircraft have been improved in a single optimization run. Frequency and the cross-orthogonality (mode shape) matrix were the primary focus for improvement, whereas other properties such as c.g. location, total weight, and off-diagonal terms of the mass orthogonality matrix were used as constraints. The end result was an improved structural dynamic finite-element model configuration for the $\mathrm{X}$-56A aircraft. Improved frequencies and mode shapes in this study increased average flutter speeds of the $\mathrm{X}-56 \mathrm{~A}$ aircraft by $7.6 \%$ compared to the baseline model.
\end{abstract}

\section{Nomenclature}

$\begin{array}{ll}A & = \\ E & = \\ f & = \\ G & = \\ I_{1} & = \\ I_{2} & = \\ J & = \\ J_{k} & = \\ K_{2} & = \\ K_{3} & = \\ K_{4} & = \\ \boldsymbol{M}_{A} & = \\ \boldsymbol{M} & = \\ \mathrm{MAC} & = \\ m & = \\ & \\ n & = \\ n_{A D} & = \\ n_{\mathrm{MD}} & = \\ \overline{\boldsymbol{S}} & = \\ V & = \\ \boldsymbol{\Phi}_{\mathrm{Aset}} & = \\ \boldsymbol{\Phi}_{g} & = \\ \boldsymbol{\Phi}_{M} & = \\ \boldsymbol{\Phi}_{S} & = \\ & \end{array}$

\author{
area of bar cross section \\ Young's modulus \\ frequency \\ shear modulus \\ area moment of inertia of bar with respect to plane 2 \\ area moment of inertia of bar with respect to plane 1 \\ objective function \\ performance indices \\ spring constant in direction 2 \\ spring constant in direction 3 \\ spring constant in direction 4 \\ analytical mass matrix obtained from Nastran \\ orthogonal matrix \\ modal assurance criteria \\ = number of modes to be use in system equivalent \\ reduction expansion process \\ $=$ number of modes for model tuning \\ $=$ number of $A$-set degrees of freedom \\ $=$ number of measured (or master) degrees of freedom \\ cross-orthogonality matrix \\ velocity \\ eigenmatrix obtained from Nastran modal analysis \\ intermediate matrix to compute $\boldsymbol{M}_{A}$ \\ eigenmatrix, corresponds to master degrees of freedom \\ eigenmatrix, corresponds to slave degrees of freedom
}

\section{Introduction}

$\mathbf{O}$

NE of the major goals of the Fundamental Aeronautics program under the NASA Aeronautics Research Mission Directorate

Presented as Paper 2014-3157 at the 15th AIAA/ISSMO Multidisciplinary Analysis and Optimization Conference, Atlanta, GA, 16-20 June 2014; received 25 June 2014; revision received 3 September 2014; accepted for publication 4 September 2014; published online 12 December 2014. This material is declared a work of the U.S. Government and is not subject to copyright protection in the United States. Copies of this paper may be made for personal or internal use, on condition that the copier pay the $\$ 10.00$ per-copy fee to the Copyright Clearance Center, Inc., 222 Rosewood Drive, Danvers, MA 01923; include the code 1533$3868 / 14$ and $\$ 10.00$ in correspondence with the CCC

*Research Manager, Aerospace Engineer, Aerostructures Branch, P.O. Box 273, Mail Stop 48201A. Senior Member AIAA.

${ }^{\dagger}$ Aerospace Engineer, Aerostructures Branch, P.O. Box 273, Mail Stop 48201A. Member AIAA.
(ARMD) is to develop a cutting-edge technology for higherperformance lighter-weight aircraft. Higher performance includes energy efficiency and operability technologies that enable advanced airframe and engine systems. Removing weight from an aircraft usually results in reduced stiffness and, therefore, increased flexibility. The increased flexibility creates an aircraft that is more susceptible to aeroelastic phenomena such as flutter, divergence, buzz, buffet, and gust response. Therefore, structural weight reduction is constrained by these adverse aeroelastic phenomena.

The ability for flexible motion control, such as gust-load alleviation and active flutter suppression, will help in opening the new design space for lighter-weight aircraft, which will increase fuel efficiency and help with noise reduction. Many active aeroelastic controller designs have been developed and proven analytically. In some cases, wind-tunnel tests have been performed to demonstrate these designed active controllers. Based on the maturation of active aeroelastic controls, it is time to perform an actual flight demonstration of these active controllers. However, there has been limited testing on active flutter suppression technology due to the high risk of vehicle loss.

An open, nonpropriety, unmanned aerial vehicle called the MultiUtility Technology Testbed (X-56A), as shown in Fig. 1, has been developed by the U.S. Air Force Research Laboratory (AFRL) for relatively high-risk aeroelastic flight demonstrations and future research. If a flight goes beyond flutter boundaries and a wing fails, then the X-56A aircraft will be fitted with a center-body-mounted ballistic parachute recovery system. Sharing the same goal to design a lighter-weight aircraft by suppressing associated aeroelastic instabilities using an active control has led to a joint collaboration between NASA and the AFRL. The AFRL provided the X-56A aircraft, ground control stations, analytical models, and ground and flight-test data to NASA. After the first flight test with flexible wings, AFRL's assets, which include two reusable center bodies, one rigid wing, three flexible wings, and ground control stations, will stay at NASA Armstrong Flight Research Center (AFRC) for future technology demonstrations, such as the NASA-designed lighter-weight wing with curvilinear spars and ribs, the Northrop Grumman-designed flying wing, and/or the Boeing-designed joined wing, as shown in Fig. 2. Furthermore, Lockheed Martin Skunk Works (LMSW) has provided NASA the finite-element models and the ground-vibration test data for this research project.

The X-56A aircraft has a wingspan of $28 \mathrm{ft}$ and a weight between 200 and $480 \mathrm{lb}$, depending on its configuration [1]. This aircraft is powered by twin JetCat P400 turbojet engines and configured for easy wing replacement. The design also includes a hard point on the 


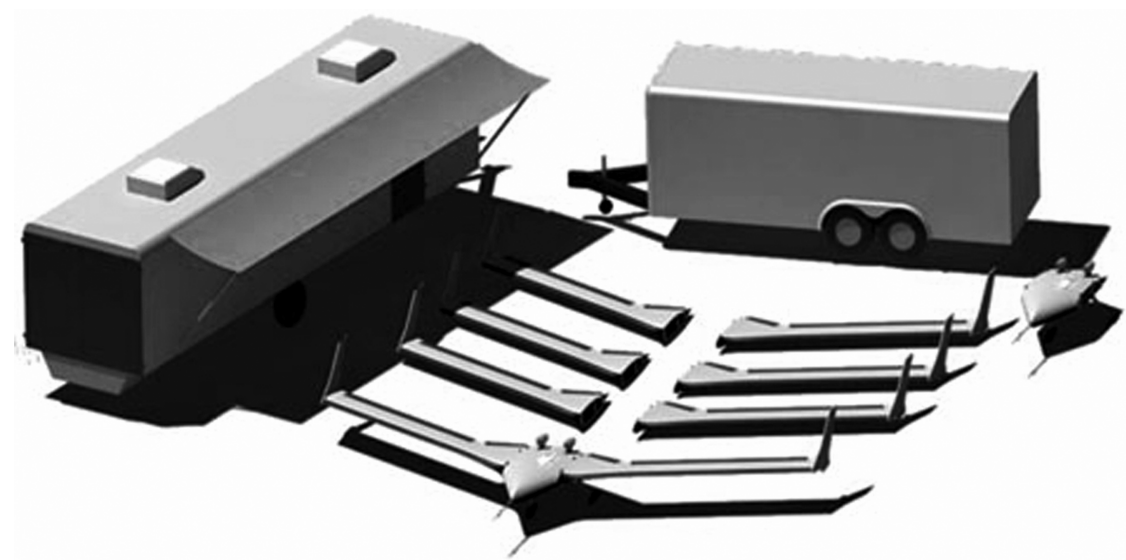

Fig. 1 X-56A aircraft with ground control stations. (Figure courtesy AFRL/Lockheed Martin).

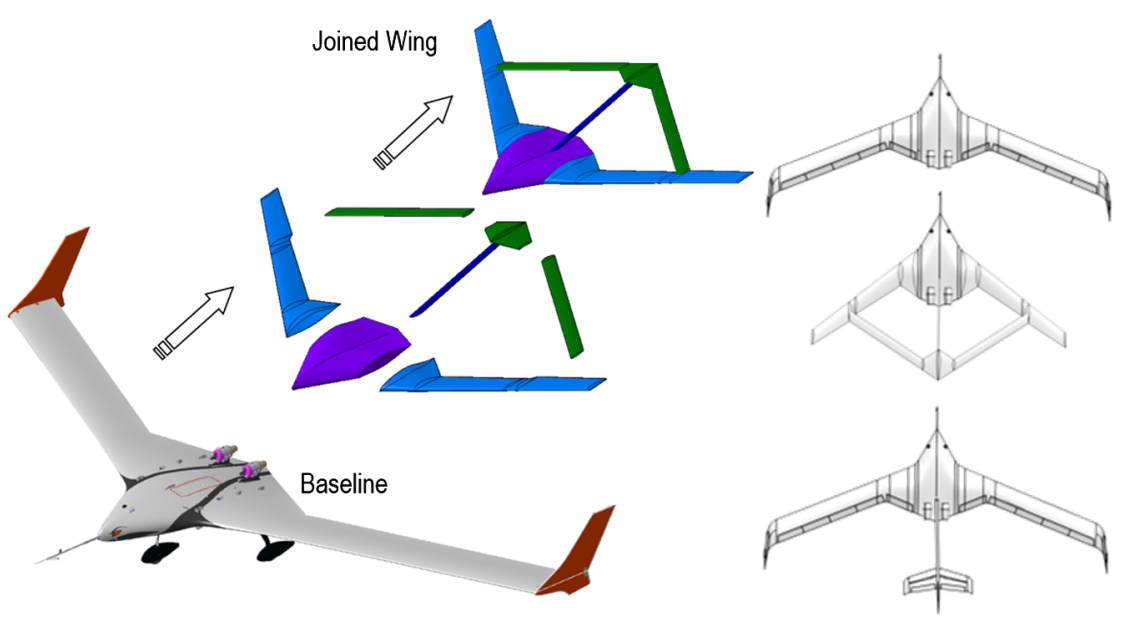

Fig. 2 Future research configurations of the X-56A aircraft.

center upper deck of the aft fuselage that can be adapted to house either a third engine or the boom for a joined wing, thereby enabling testing of more advanced aerodynamic concepts. A ground-vibration test was completed on 17 December 2012, and test data have been provided to NASA for model validations.

Modern aircraft make extensive use of composite materials to reduce weight. Aircraft aeroservoelastic models have significant levels of model parameter uncertainty due to the composite manufacturing process. In an attempt to mitigate this, ground-vibration test (GVT) data can be used to tune a model and help to improve its accuracy over a wider range of operating environments. This tuning and adaption procedure has become an accepted and necessary step in making models more reliable; however, it cannot handle or eliminate all uncertainties in a finite-element (FE) model. To minimize the uncertainties in aeroelastic as well as aeroservoelastic response computations, the structural dynamic model, the unsteady aerodynamic model, and the actuator model should be validated with respect to the corresponding test data [2,3]. Small modeling errors in an FE model will eventually induce errors in the structural flexibility and mass, thus propagating into unpredictable errors in the unsteady aerodynamics and the control law design. Tuning an FE model using measured data to minimize the modeling uncertainties is a challenging task in the area of structural dynamics.

Over the past few decades, major progress has been made to develop methods to update these finite-element models to match computed frequencies and mode shapes to the corresponding measured GVT data [4-8]. Recently, attention has shifted toward applying these methods to the health monitoring of structures to address this problem [9-12]. Literature reviews on finite-element model updating methods are detailed in [13-15]. These methods can be separated into two different categories: direct methods and indirect (or parametric) methods. The direct methods correct the mass and stiffness matrices but do not take into account the structure's physical characteristics. On the other hand, the indirect (or parametric) methods correct the model by altering the physical structural parameters, which include (but are not limited to) structural sizing properties, point mass and spring properties, and material properties. In this study, the parametric method was selected for the modeltuning optimization process.

Supporting the ARMD guidelines, NASA AFRC has developed a computer code for FE model tuning [2,16] using the object-oriented optimization $\left(\mathrm{O}^{3}\right)$ tool [17] together with Nastran [18], an FE analysis computer software program. Three optimizer algorithms have been incorporated into the $\mathrm{O}^{3}$ tool: design optimization tools (DOTs) [19], based on a gradient-based algorithm; the genetic algorithm $(\overline{\mathrm{GA}})$ [20], which is a class of stochastic and global optimization; and bigbang-big-crunch algorithms [21-24]. This $\mathrm{O}^{3}$ tool, as shown in Fig. 3 , was crucial in the development of a multidisciplinary design, analysis, and optimization tool [25] and an unsteady aerodynamic model-tuning tool [3].

The primary objective of this study is to obtain the GVT-validated structural dynamic finite-element model of the X-56A aircraft. Based on the validated model, flutter boundaries are computed again and compared with results obtained from the nonvalidated model. The secondary objective of this study is to improve and extend the previously developed structural dynamic FE model-tuning tool [2] and demonstrate this tool using the $\mathrm{X}-56 \mathrm{~A}$ aircraft as a sample case. 


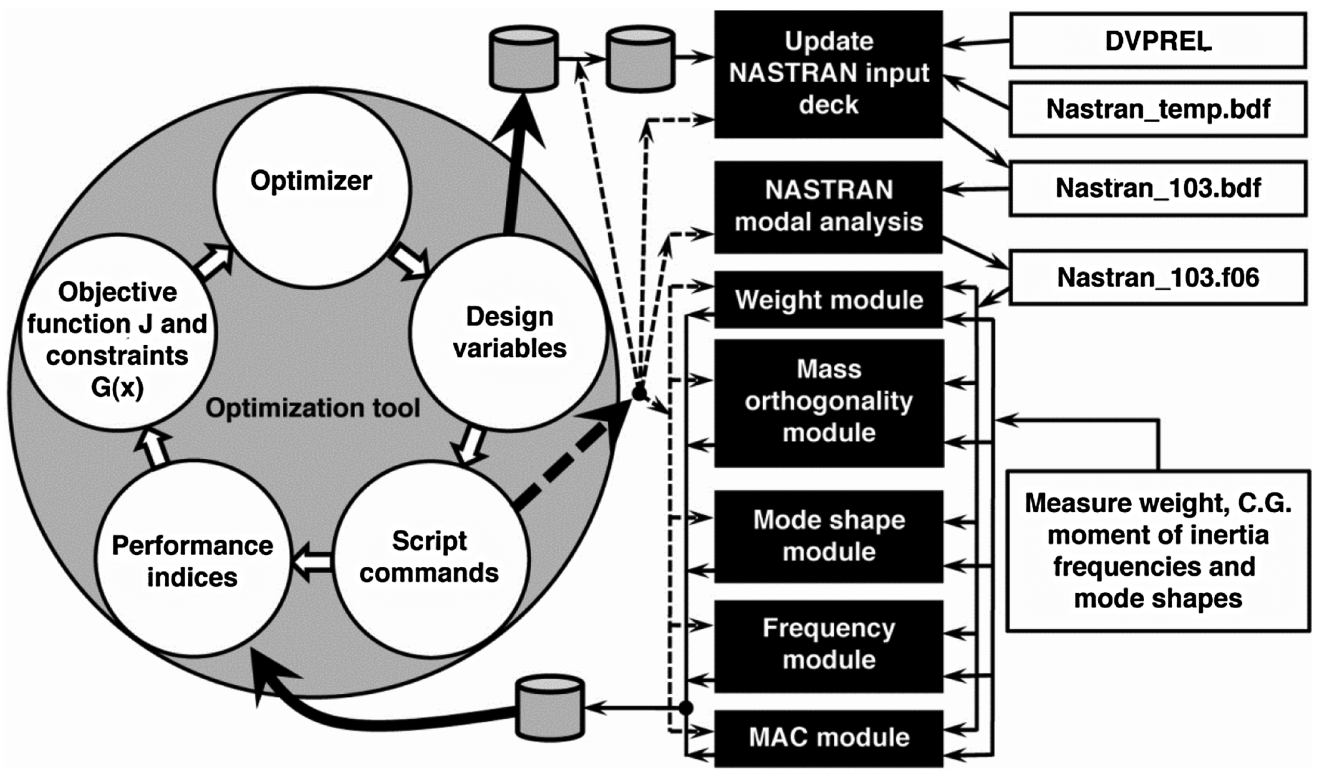

Fig. 3 Structural dynamic model tuning based on the $\mathrm{O}^{3}$ tool.

\section{Optimization Theoretical Background}

In the FE model-tuning tool, the user chooses an optimization algorithm, defines the continuous as well as the discrete design variables with side constraints, starting values, and scaling factors, and provides script and data file names for each performance index. Communication between the $\mathrm{O}^{3}$ tool and each analysis submodule is performed through the use of design variables and performance indices. Once the $\mathrm{O}^{3}$ tool is executed, design variables are created and saved in an external data file. This data file cannot be shared with other analysis submodules; therefore, a script command makes a copy of this data file first. Script commands for each performance index perform the following tasks.

1) Prepare data for analysis program (preprocessor module).

2) Submit analysis job (analyzer module)

3) Read result files, compute the required performance index value, and save it (postprocessor module).

Analysis submodules of the extended model-tuning tool are explained in this section. Blocks in Fig. 3 can be categorized into three modules: the preprocessor, the analyzer, and the postprocessor modules. The preprocessor module (update Nastran input deck block) in Fig. 3 is used to create and update input files based on the design variable values provided by the $\mathrm{O}^{3}$ tool before executing the analyzer module. The analyzer module (Nastran modal analysis block) in Fig. 3 can be a commercial and/or the in-house codes for a specific discipline. The script commands will execute the analyzer module automatically. The postprocessor module is used to postprocess the Nastran output file, which is computed from the analyzer module, and it automatically computes the performance indices. Several submodules were developed for the structural dynamic FE model tuning.

\section{A. Weight Submodule}

A weight generator in Nastran code and measured test data are used to compute the maximum 10 performance indices associated with the total weight, c.g. locations, and the moments of inertia. Note that the $G$ in the subscripts for the following variables displayed represents the test-measured data value as shown in Eqs. (1-10):

$$
\begin{aligned}
& J_{1}=\left(\frac{W-W_{G}}{W_{G}}\right)^{2} \text { (total weight) } \\
& J_{2}=\left(\frac{X-X_{G}}{X_{G}}\right)^{2}(x \text { c.g. location })
\end{aligned}
$$

$$
\begin{aligned}
& J_{3}=\left(\frac{Y-Y_{G}}{Y_{G}}\right)^{2}(y \text { c.g. location }) \\
& J_{4}=\left(\frac{Z-Z_{G}}{Z_{G}}\right)^{2}(z \text { c.g. location }) \\
& J_{5}=\left(\frac{I_{x x}-I_{x x G}}{I_{x x G}}\right)^{2}\left(I_{x x} \text { at c.g. }\right) \\
& J_{6}=\left(\frac{I_{y y}-I_{y y G}}{I_{y y G}}\right)^{2}\left(I_{y y} \text { at c.g. }\right) \\
& J_{7}=\left(\frac{I_{z z}-I_{z z G}}{I_{z z G}}\right)^{2}\left(I_{z z} \text { at c.g. }\right) \\
& J_{10}=\left(\frac{I_{z x}-I_{z x G}}{I_{z x G}}\right)^{2}\left(I_{z x} \text { at c.g. }\right) \\
& J_{8}=\left(\frac{I_{x y}-I_{x y G}}{I_{x y G}}\right)^{2}\left(I_{x y} \text { at c.g. }\right)
\end{aligned}
$$

\section{B. Mass Orthogonality Submodule}

This submodule computes each off-diagonal term of the orthogonality matrix using Eq. (11):

$$
\overline{\boldsymbol{M}}=\boldsymbol{\Phi}_{G \text { Aset }}^{T} \boldsymbol{M}_{A} \boldsymbol{\Phi}_{G \text { Aset }}(\text { order }=n \times n)
$$

In Eq. (11), the order of a reduced-order mass matrix $\boldsymbol{M}_{\boldsymbol{A}}$, is based on the $A$-set degrees of freedom [18] and computed using the system 
equivalent reduction expansion process (SEREP) shown in Eqs. (12) and (13) [26]:

$$
\begin{gathered}
\boldsymbol{M}_{A}=\boldsymbol{\Phi}_{g}^{T} \boldsymbol{\Phi}_{g}\left(\text { order }=n_{A D} \times n_{A D}\right) \\
\boldsymbol{\Phi}_{g}=\left(\boldsymbol{\Phi}_{\text {Aset }}^{T} \boldsymbol{\Phi}_{\text {Aset }}\right)^{-1} \boldsymbol{\Phi}_{\text {Aset }}^{T}\left(\text { order }=m \times n_{A D}\right)
\end{gathered}
$$

where the matrix $\boldsymbol{\Phi}_{\text {Aset }}$ (order $=n_{A D} \times m$ ) is an eigenmatrix obtained from Nastran modal analysis, and $m$ and $n_{A D}$ are the number of modes to be use in the SEREP and the number of $A$-set degrees of freedom, respectively. The expanded GVT mode shape $\boldsymbol{\Phi}_{G \text { Aset }}$, is also computed using SEREP as follows.

Reorder the matrix $\boldsymbol{\Phi}_{\text {Aset }}$, then $\boldsymbol{\Phi}_{\text {Aset }} \equiv\left[\begin{array}{c}\boldsymbol{\Phi}_{M} \\ \boldsymbol{\Phi}_{S}\end{array}\right]$, and the matrix $\boldsymbol{\Phi}_{G \text { Aset }}\left(\right.$ order $\left.=n_{A D} \times n\right)$ will become Eq. (14):

$$
\boldsymbol{\Phi}_{G \text { Aset }}=\left[\begin{array}{l}
\boldsymbol{\Phi}_{M}\left(\boldsymbol{\Phi}_{M}^{T} \boldsymbol{\Phi}_{M}\right)^{-1} \boldsymbol{\Phi}_{M}^{T} \\
\boldsymbol{\Phi}_{S}\left(\boldsymbol{\Phi}_{M}^{T} \boldsymbol{\Phi}_{M}\right)^{-1} \boldsymbol{\Phi}_{M}^{T}
\end{array}\right] \tilde{\boldsymbol{\Phi}}_{G M}
$$

where matrices $\quad \boldsymbol{\Phi}_{M} \quad$ (order $\left.=n_{A D} \times m\right)$ and $\quad \boldsymbol{\Phi}_{S} \quad$ [order $=$ $\left.\left.\left(n_{A D}-n_{\mathrm{MD}}\right) \times m\right)\right]$ are the master and slave eigenmatrices. The $n_{\mathrm{MD}}$ and $n$ are the measured (or master) degrees of freedom and the number of modes for model tuning, respectively; and $\tilde{\boldsymbol{\Phi}}_{G M}\left(n_{\mathrm{MD}} \times n\right)$ is the measured eigenmatrix obtained from GVT.

The number of performance indices (off-diagonal term square of matrix $\bar{M}$ ),

$$
J_{k}=\bar{M}_{i j}^{2} \quad i \neq j \quad i \& j=1,2, \ldots, n
$$

computed in this submodule is

$$
L_{M}=\frac{n^{2}-n}{2}
$$

where $k=11, \ldots, 10+L_{M}$. In the previous version of modeltuning tool, only one performance index,

$$
J=\sum_{i=1}^{n} \sum_{j=i+1}^{n} \overline{\boldsymbol{M}}_{i j}^{2}
$$

was defined for mass orthogonality.

The extended version of the model-tuning tool required a significant number of performance indices because individual offdiagonal terms, as shown in Eqs. (15) and (16), were performance indices instead of using a single norm value of off-diagonal terms as in Eq. (17), which was used in the previous version of the modeltuning tool $[2,16]$. With the extended version, it was much easier to improve each off-diagonal term element as opposed to the previous version.

\section{Frequency Submodule}

Performance indices associated with the $i$ th frequency error are computed from Eq. (18):

$$
J_{k}=\left(\frac{f_{i}-f_{i G}}{f_{i G}}\right)^{2} \quad k=11+L_{M}, \ldots, 10+L_{M}+m
$$

where $f_{i}$ and $f_{i G}$ are the $i$ th numerical and measured frequencies. The $m$ performance indices (number of modes to be matched) are computed in this submodule. Similarly, the previous version of the model-tuning tool had only one performance index for frequency error and was defined as Eq. (19):

$$
J=\sum_{i=1}^{n}\left(\frac{f_{i}-f_{i G}}{f_{i G}}\right)^{2}
$$

\section{Mode Shape Submodule}

In the previous version of the model-tuning tool, numerical mode shapes were improved to minimize mode shape error at sensor locations using a single performance index. In the extended version, each off-diagonal term of the cross-orthogonality matrix is computed using Eq. (20):

$$
\overline{\boldsymbol{S}}=\boldsymbol{\Phi}_{G \text { Aset }}^{T} \boldsymbol{M}_{A} \boldsymbol{\Phi}_{\text {Aset }}
$$

The number of performance indices (off-diagonal term square of matrix $\overline{\boldsymbol{S}})$ in Eq. (21):

$$
J_{k}=\bar{S}_{i j}^{2} \quad i \neq j \quad i \& j=1,2, \ldots, n
$$

computed in this submodule is shown in Eq. (22):

$$
L_{s}=n^{2}-n
$$

where $n$ is the order of the cross-orthogonality mass matrix $\bar{S}$, and $k=11+L_{M}+m, \ldots, 10+L_{M}+m+L_{s}$.

\section{E. Modal Assurance Criteria Submodule}

In this submodule, a modal assurance criteria (MAC) matrix MAC $i j$, defined as Eq. (르),

$$
\operatorname{MAC}_{i j}=\frac{\left(\sum_{k=1}^{n} \Phi_{k i G}^{T} \Phi_{k j}\right)^{T}}{\left(\sum_{k=1}^{n} \Phi_{k i G}^{T} \Phi_{k i G}\right)\left(\sum_{k=1}^{n} \Phi_{k j}^{T} \Phi_{k j}\right)}
$$

is used to compute the corresponding performance indices. The diagonal terms of the matrix $\mathrm{MAC}_{i j}$ are selected as $m$ performance indices shown in Eq. (24):

$$
J_{k}=\mathrm{MAC}_{i i}
$$

where $i=1, \ldots, m$ and, $k+11+L_{M}+m+L_{s}, \ldots, 10+L_{M}+$ $2 m+L_{s}$.

The optimization problem statement can be written as

minimize $J=\sum_{i} w_{i} J_{i}$ (performance index $i$ selected for objective functions) such that $J_{k} \leq \varepsilon_{k}$ (performance index $k$ selected for constraint functions)

A constrained optimization problem in Eq. (25) can be solved using the $\mathrm{O}^{3}$ tool. In Eq. (25), $\varepsilon_{k}$ and $w_{i}$ are small values that can be adjusted according to the upper limit values of constraints and the $i$ th weighting factor of the performance index $J_{i}$, respectively.

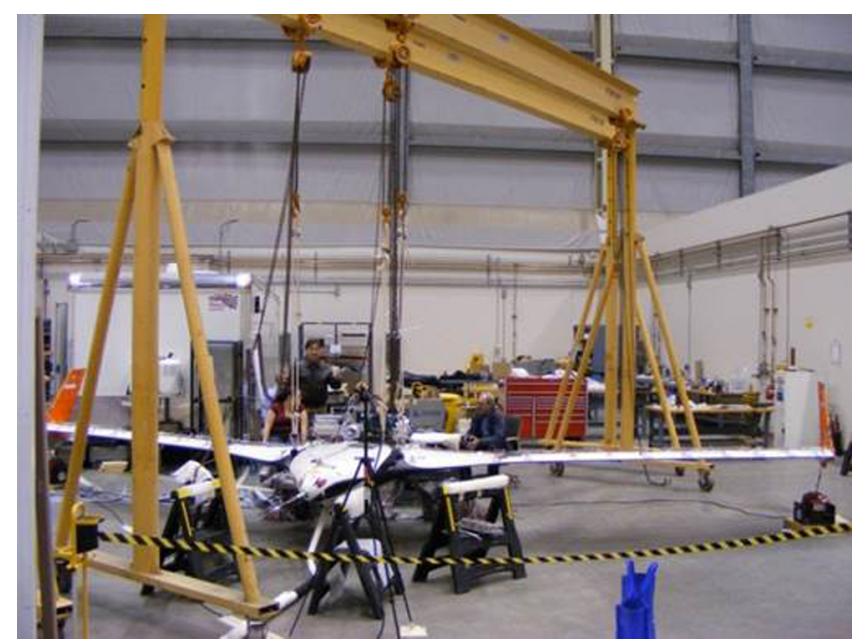

Fig. 4 X-56A aircraft with soft suspension system. (Photo courtesy AFRL/Lockheed Martin). 
Table 1 Measured and computed flexible modes of the X-56A aircraft

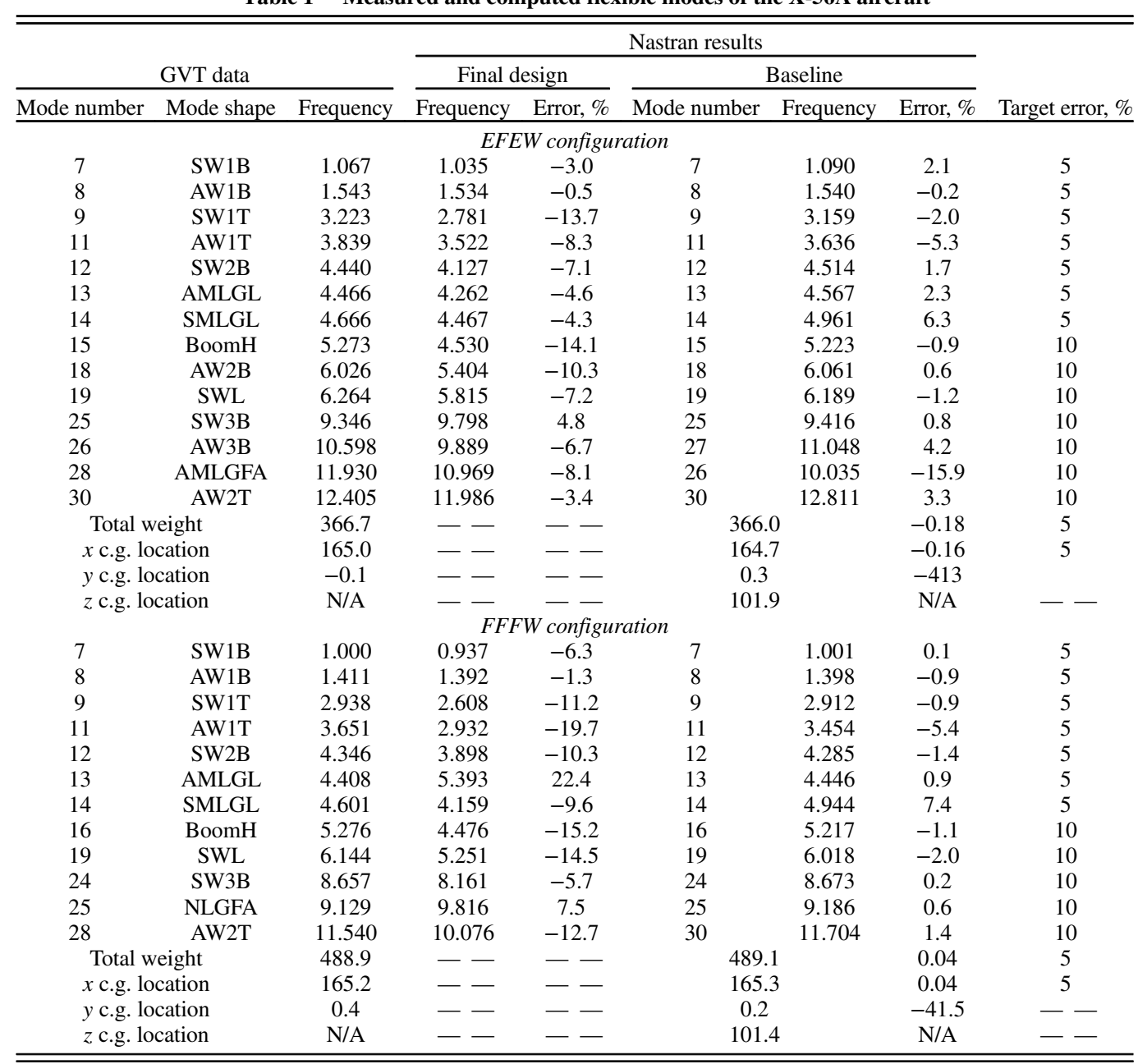

\section{Ground-Vibration Tests}

The GVT of the X-56A aircraft with a flexible wing configuration was completed by ATA Engineering, Inc., under the supervision of LMSW, on 17 December 2012. The free-free boundary condition with a soft suspension system, as shown in Fig. 4, was used in this

Table 2 Acronyms for the description of the mode shape

\begin{tabular}{ll}
\hline \hline Acronym & \multicolumn{1}{c}{ Mode shape } \\
\hline AMLGFA & Antisymmetric main landing gear forward and aft \\
AMLGL & Antisymmetric main landing gear lateral \\
AW1B & Antisymmetric wing first bending \\
AW1T & Antisymmetric wing first torsion \\
AW2B & Antisymmetric wing second bending \\
AW2T & Antisymmetric wing second torsion \\
AW3B & Antisymmetric wing third bending \\
AWFA & Antisymmetric wing forward and aft \\
AWL & Antisymmetric winglet \\
BoomH & Boom horizontal \\
BoomV & Boom vertical \\
NLGFA & Nose landing gear forward and aft \\
NLGL & Nose landing gear lateral \\
SMLGFA & Symmetric main landing gear forward and aft \\
SMLGL & Symmetric main landing gear lateral \\
SWL & Symmetric winglet \\
SW1B & Symmetric wing first bending \\
SW1T & Symmetric wing first torsion \\
SW2B & Symmetric wing second bending \\
SW2T & Symmetric wing second torsion \\
SW3B & Symmetric wing third bending \\
SWFA & Symmetric wing forward and aft \\
\hline \hline
\end{tabular}

test. The assembled X-56A aircraft with flexible wings was tested in two weight configurations: 1) empty fuel, empty water (EFEW), and 2) full fuel, full water (FFFW) [1]. It should be noted that, during ground vibration and flight testing, water was used to simulate fuel weight in the wings. Nondimensional frequency results are summarized in Table 1 . All the frequencies in this paper were nondimensionalized with respect to the first measured frequency obtained from GVT with the FFFW configuration. A total of 120 degrees of freedom have been measured during the GVT. This measured mode shape information was expanded to the total of 1477 $A$-set degrees of freedom using Eq. (14), and mode shape information was computed from Nastran modal analysis of the LMSW's final design model and baseline model with both the EFEW and FFFW configurations. A total of the first 30 modes including the six rigid body modes were used in this mode shape fitting. In this study, the final design model and the baseline model are defined as the nonvalidated and the test-validated FE models, respectively. The test-validated FE model in Table 1 for the EFEW and the FFFW configurations were created by LM $\overline{S W}$. Acronyms for the description of the mode shape are given in Table 2 . The baseline model will be the starting configuration of the model-tuning procedure. Figure 5 shows how the models are defined in this study.

\section{Pretuning Analysis}

The GVTs of the X-56A aircraft were performed for the verification of the FE model. The following modal survey requirements are observed in $[27,28]$.

Military standard:

1) Analytical model frequencies are to be within $3 \%$ of test frequencies [27]. 


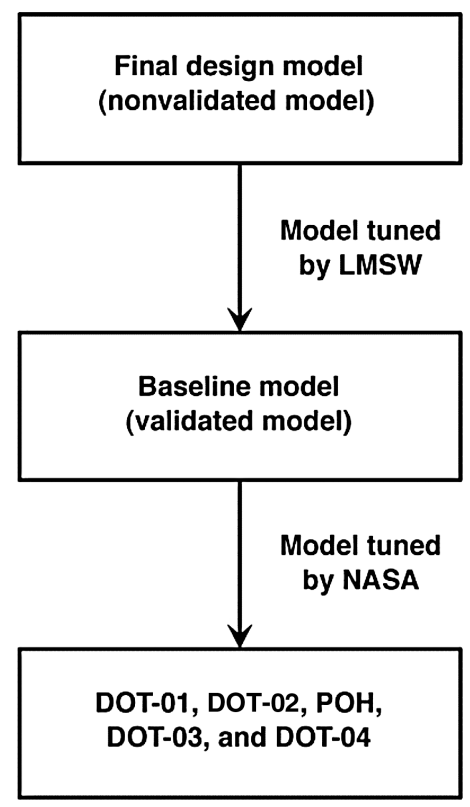

Fig. 5 Different models in this study.

2) Using a cross-orthogonality matrix formed from the analytical mass matrix and the analytical and test modes, corresponding modes are to exhibit at least $95 \%$ correlation, and dissimilar modes are to be orthogonal to within $10 \%$ [27].

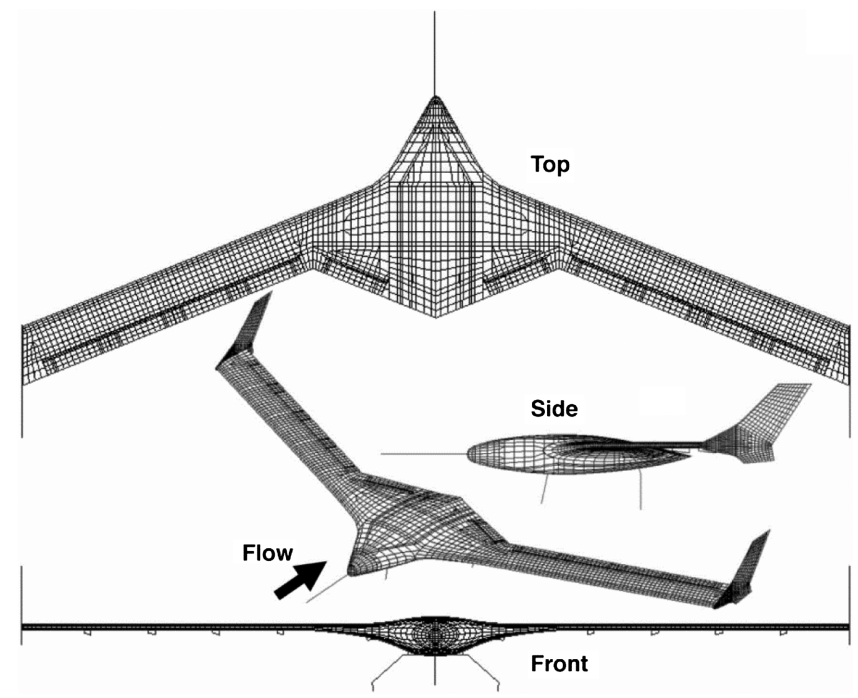

Fig. 6 Finite-element model of the X-56A aircraft.

NASA standard:

1) Agreement between test and analysis natural frequencies shall, as a goal, be within 5\% for the significant modes [28].

2) Accurate mass representation of the test article shall be demonstrated with orthogonality checks using the analytical mass matrix $\mathbf{M}$ and the test mode shapes $\boldsymbol{\Phi}_{G}$. The orthogonality matrix is

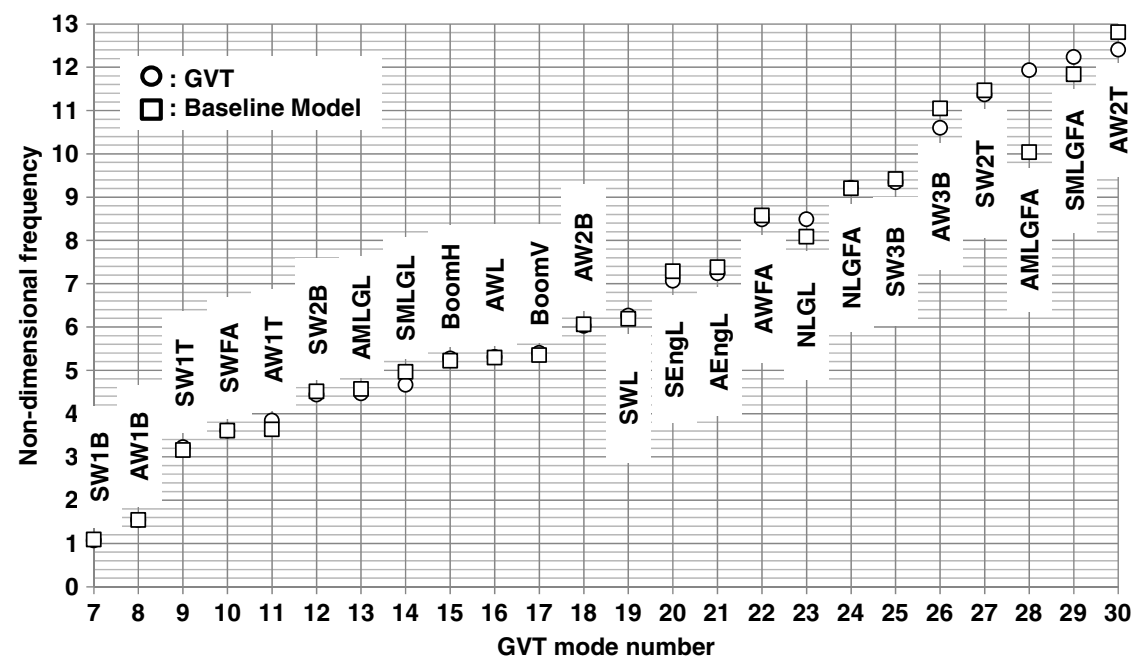

Fig. 7 Comparisons of nondimensional frequencies (EFEW case).

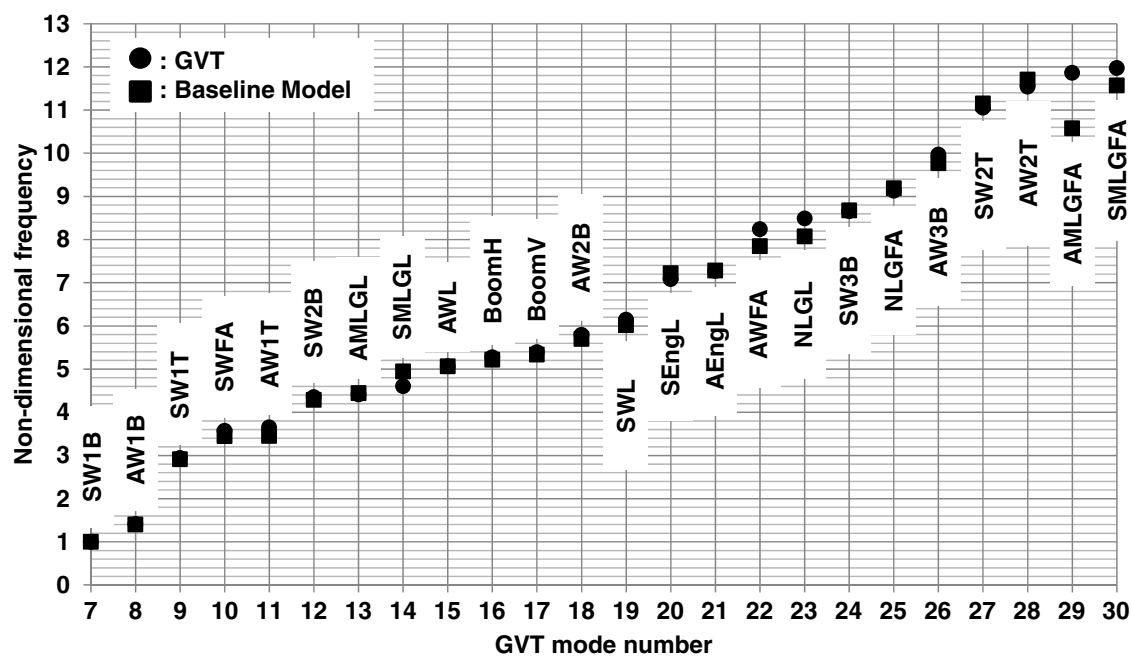

Fig. 8 Comparisons of nondimensional frequencies (FFFW case). 


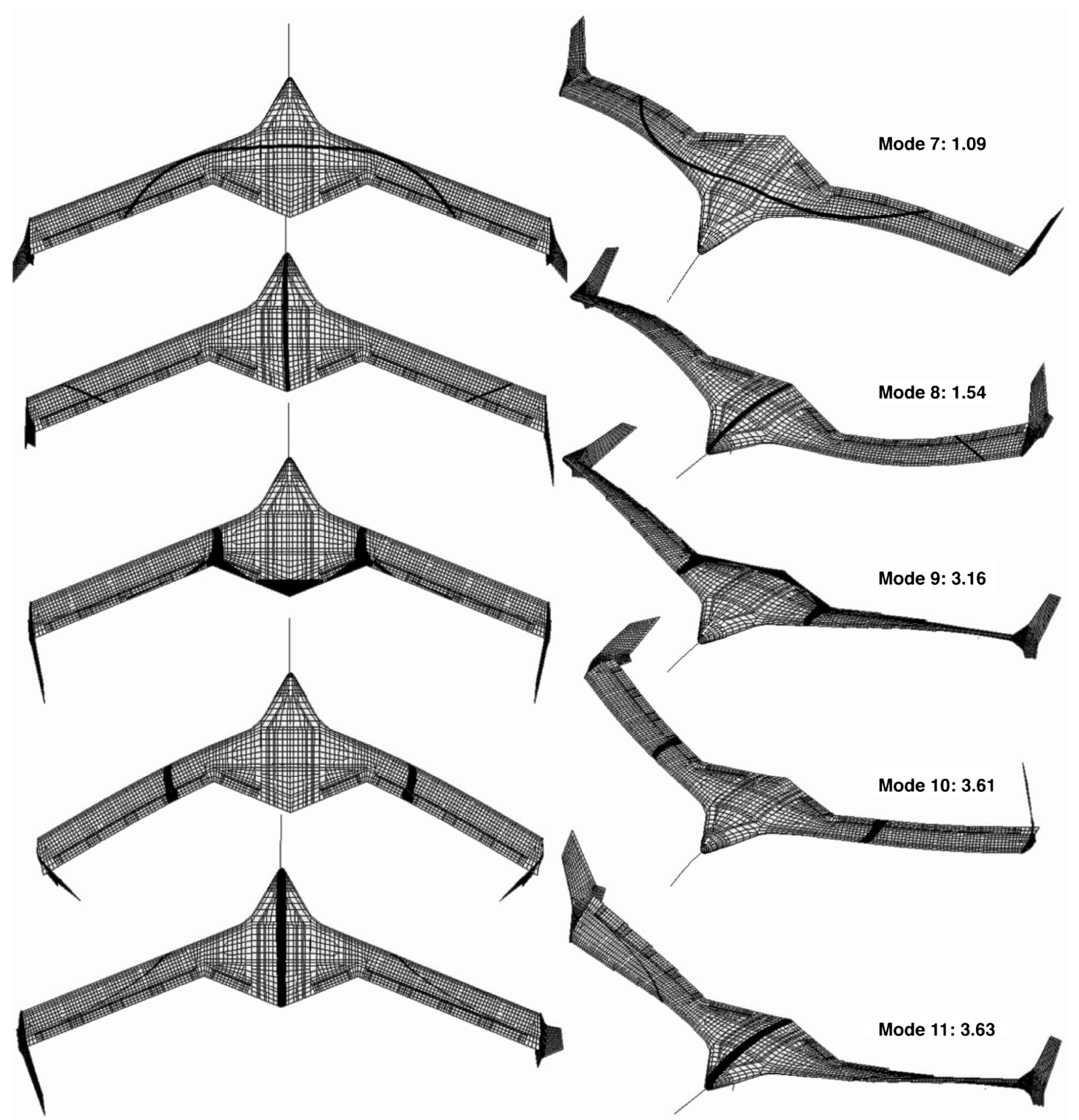

Fig. 9 First five flexible modes with the EFEW configuration.

\begin{tabular}{|c|c|c|c|c|c|c|c|c|}
\hline \multicolumn{9}{|c|}{ EFEW configuration } \\
\hline & Mode shape & SW1B & AW1B & SW1T & AW1T & SW2B & AMLGL & SMLGL \\
\hline Mode shape & Mode number & $7^{*}$ & 8 & 9 & 11 & 12 & 13 & 14 \\
\hline SW1B & $7^{* *}$ & $\mathbf{0 . 9 9}$ & 0.00 & 0.00 & 0.00 & 0.08 & 0.00 & 0.01 \\
\hline AW1B & 8 & 0.00 & $\mathbf{1 . 0 0}$ & 0.00 & 0.00 & 0.00 & 0.09 & 0.00 \\
\hline SW1T & 9 & 0.01 & 0.00 & $\mathbf{0 . 9 1}$ & 0.00 & 0.01 & 0.00 & 0.01 \\
\hline AW1T & 11 & 0.00 & 0.01 & 0.00 & $\mathbf{0 . 9 7}$ & 0.00 & 0.16 & 0.00 \\
\hline SW2B & 12 & 0.09 & 0.00 & 0.09 & 0.01 & $\mathbf{0 . 9 5}$ & 0.02 & 0.27 \\
\hline AMLGL & 13 & 0.00 & 0.06 & 0.00 & 0.43 & 0.03 & $\mathbf{0 . 9 3}$ & 0.02 \\
\hline SMLGL & 14 & 0.05 & 0.00 & 0.07 & 0.00 & 0.93 & 0.00 & $\mathbf{0 . 5 1}$ \\
\hline \multicolumn{7}{|c|}{ FFFW configuration } & & \\
\hline Mode shape & Mode number & $7 *$ & 8 & 9 & 11 & 12 & 13 & 14 \\
\hline SW1B & $7 * *$ & $\mathbf{0 . 9 9}$ & 0.00 & 0.02 & 0.00 & 0.08 & 0.00 & 0.01 \\
\hline AW1B & 8 & 0.00 & $\mathbf{1 . 0 0}$ & 0.00 & 0.01 & 0.00 & 0.07 & 0.00 \\
\hline SW1T & 9 & 0.04 & 0.00 & $\mathbf{0 . 9 3}$ & 0.00 & 0.01 & 0.00 & 0.02 \\
\hline AW1T & 11 & 0.00 & 0.04 & 0.00 & $\mathbf{0 . 9 0}$ & 0.01 & 0.09 & 0.01 \\
\hline SW2B & 12 & 0.07 & 0.00 & 0.06 & 0.00 & $\mathbf{0 . 9 9}$ & 0.00 & 0.14 \\
\hline AMLGL & 13 & 0.00 & 0.04 & 0.00 & 0.37 & 0.01 & $\mathbf{0 . 9 6}$ & 0.01 \\
\hline SMLGL & 14 & 0.03 & 0.00 & 0.04 & 0.00 & 0.84 & 0.02 & $\mathbf{0 . 3 8}$ \\
\hline
\end{tabular}

*: Nastran mode number (Nastran mode extraction order: $1-6=$ rigid body $\&$ higher than $7=$ flexible)

**: GVT mode number (GVT mode extraction order: $1-6=$ rigid body $\&$ higher than $7=$ flexible) 


\begin{tabular}{|c|c|c|c|c|c|c|c|c|}
\hline \multicolumn{9}{|c|}{ EFEW configuration } \\
\hline & Mode shape & SW1B & AW1B & SW1T & AW1T & SW2B & AMLGL & SMLGL \\
\hline Mode shape & Mode number & $7^{* *}$ & 8 & 9 & 11 & 12 & 13 & 14 \\
\hline SW1B & $7^{* *}$ & $\mathbf{1 . 0 0 0}$ & -0.021 & -0.054 & -0.011 & 0.026 & 0.024 & -0.033 \\
\hline AW1B & 8 & -0.021 & $\mathbf{1 . 0 0 0}$ & 0.012 & 0.002 & 0.004 & 0.040 & 0.002 \\
\hline SW1T & 9 & -0.054 & 0.012 & $\mathbf{1 . 0 0 0}$ & 0.004 & 0.035 & -0.007 & -0.025 \\
\hline AW1T & 11 & -0.011 & 0.002 & 0.004 & $\mathbf{1 . 0 0 0}$ & 0.022 & -0.093 & 0.003 \\
\hline SW2B & 12 & 0.026 & 0.004 & 0.035 & 0.022 & $\mathbf{1 . 0 0 0}$ & -0.143 & 0.143 \\
\hline AMLGL & 13 & 0.024 & 0.040 & -0.007 & -0.093 & -0.143 & $\mathbf{1 . 0 0 0}$ & 0.006 \\
\hline SMLGL & 14 & -0.033 & 0.002 & -0.025 & 0.003 & 0.143 & 0.006 & $\mathbf{1 . 0 0 0}$ \\
\hline \hline \multicolumn{7}{|c|}{ FFFW configuration } \\
\hline Mode shape & Mode shape & SW1B & AW1B & SW1T & AW1T & SW2B & AMLGL & SMLGL \\
\hline SW1B & $7 * *$ & $7^{* *}$ & 8 & 9 & 11 & 12 & 13 & 14 \\
\hline AW1B & 8 & $\mathbf{1 . 0 0 0}$ & 0.013 & -0.048 & 0.014 & 0.019 & 0.008 & -0.066 \\
\hline SW1T & 9 & -0.013 & $\mathbf{1 . 0 0 0}$ & -0.010 & 0.013 & -0.005 & -0.062 & -0.011 \\
\hline AW1T & 11 & -0.048 & -0.010 & $\mathbf{1 . 0 0 0}$ & -0.019 & 0.007 & -0.013 & -0.028 \\
\hline SW2B & 12 & 0.019 & -0.005 & 0.007 & -0.026 & $\mathbf{1 . 0 0 0}$ & 0.003 & 0.150 \\
\hline AMLGL & 13 & 0.008 & -0.062 & -0.013 & 0.093 & 0.003 & $\mathbf{1 . 0 0 0}$ & -0.077 \\
\hline SMLGL & 14 & -0.066 & -0.011 & -0.028 & 0.017 & 0.150 & -0.077 & $\mathbf{1 . 0 0 0}$ \\
\hline
\end{tabular}

**: GVT mode number

Fig. 11 Orthogonality matrix of the baseline model.

computed as $\boldsymbol{\Phi}_{G}^{T} \mathbf{M} \boldsymbol{\Phi}_{G}$. As a goal, the off-diagonal terms of the orthogonality matrix should be less than 0.1 for significant modes based on the diagonal terms normalized to 1.0 [28].

3) Mode shape comparisons shall be required via crossorthogonality checks using the test modes $\boldsymbol{\Phi}_{G}$, the analytical modes $\boldsymbol{\Phi}_{A}$, and the analytical mass matrix $\mathbf{M}$. The cross-orthogonality matrix is computed as $\boldsymbol{\Phi}_{G}^{T} \mathbf{M} \boldsymbol{\Phi}_{A}$. As a goal, the absolute value of the cross-orthogonality between corresponding test and analytical mode shapes should be greater than 0.9 , and all other terms of the matrix should be less than 0.1 for all significant modes. Additionally, qualitative comparisons between test modes and analytical modes using mode shape animation and/or deflection plots shall be performed [28].

Modal analyses of the X-56A aircraft with the EFEW and FFFW weight configurations are performed and summarized in the following sections.

\section{A. Modal Analysis}

For the structural dynamic FE model tuning, the EFEW and FFFW weight configurations are selected in this study. A structural dynamic FE model of the X-56A aircraft, with a total of 8262 nodes, for Nastran modal analysis is shown in Fig. 6. The most important frequencies of the X-56A aircraft are summarized in Table 1 as well as Figs. 7, 8. Mode shapes of the first five flexible modes with the EFEW configuration are shown in Fig. 9.

The symmetric and antisymmetric engine lateral modes were not captured numerically in this modal analysis with the final design model due to the fact that the engines are connected to the center body through the use of the rigid bar elements in MSC/Nastran. It should be noted that surprisingly large frequency errors are observed with the final design model, as shown in Table 1 . Frequency errors for the final design model were improved when LMSW performed their model tuning. As shown in Table 1, frequency errors of both the EFEW and FFFW configurations decreased significantly after LMSW's model tuning, except for a few modes. Frequency errors of mode numbers 11 and 14 for the EFEW and FFFW configurations and 28 for the EFEW configuration are still larger than 5 and $10 \%$, respectively.

The MAC matrices of the baseline X-56A model with the EFEW and FFFW configurations are shown in Fig. 10. In general, diagonal terms of the MAC matrices are acceptable because they are above $90 \%$, with the exception of mode number 14 , the symmetric main landing gear lateral (SMLGL) mode, for the FFFW configuration.

The orthogonality and cross-orthogonality matrices of the baseline model with the EFEW and FFFW configurations are given in Figs. 11,

\begin{tabular}{|c|c|c|c|c|c|c|c|c|}
\hline \multicolumn{9}{|c|}{ EFEW configuration } \\
\hline & Mode shape & SW1B & AW1B & SW1T & AW1T & SW2B & AMLGL & SMLGL \\
\hline Mode shape & Mode number & $7^{*}$ & 8 & 9 & 11 & 12 & 13 & 14 \\
\hline SW1B & $7^{* *}$ & $\mathbf{- 1 . 0 0 0}$ & -0.015 & -0.032 & 0.006 & 0.026 & 0.012 & -0.074 \\
\hline AW1B & 8 & 0.005 & $\mathbf{1 . 0 0 0}$ & 0.004 & 0.015 & 0.005 & 0.031 & 0.003 \\
\hline SW1T & 9 & 0.028 & 0.007 & $\mathbf{1 . 0 0 0}$ & -0.021 & -0.151 & -0.004 & -0.024 \\
\hline AW1T & 11 & 0.000 & 0.016 & 0.003 & $-\mathbf{1 . 0 0 0}$ & 0.010 & 0.095 & -0.002 \\
\hline SW2B & 12 & 0.000 & 0.005 & 0.162 & -0.026 & $\mathbf{1 . 0 0 0}$ & -0.244 & -0.451 \\
\hline AMLGL & 13 & -0.009 & 0.013 & 0.020 & 0.211 & 0.133 & $\mathbf{1 . 0 0 0}$ & -0.004 \\
\hline SMLGL & 14 & -0.011 & 0.003 & 0.098 & 0.006 & 0.618 & -0.076 & $\mathbf{1 . 0 0 0}$ \\
\hline \hline \multicolumn{7}{|c|}{ FFFW configuration } & & \\
\hline Mode shape & Mode number & SW1B & AW1B & SW1T & AW1T & SW2B & AMLGL & SMLGL \\
\hline SW1B & $7 * *$ & 8 & 9 & 11 & 12 & 13 & 14 \\
\hline AW1B & 8 & $\mathbf{1 . 0 0 0}$ & -0.007 & 0.032 & 0.009 & 0.033 & 0.011 & 0.064 \\
\hline SW1T & 9 & -0.023 & 0.004 & $-\mathbf{1 . 0 0 0}$ & -0.018 & -0.133 & -0.005 & 0.016 \\
\hline AW1T & 11 & -0.018 & -0.004 & 0.029 & $\mathbf{1 . 0 0 0}$ & 0.014 & -0.109 & -0.009 \\
\hline SW2B & 12 & -0.010 & 0.003 & -0.132 & -0.003 & $\mathbf{1 . 0 0 0}$ & -0.052 & 0.220 \\
\hline AMLGL & 13 & -0.003 & 0.008 & -0.004 & 0.208 & 0.070 & $\mathbf{1 . 0 0 0}$ & 0.003 \\
\hline SMLGL & 14 & -0.013 & 0.011 & -0.049 & 0.012 & 0.366 & -0.121 & $\mathbf{- 1 . 0 0 0}$ \\
\hline
\end{tabular}

*: Nastran mode number

**: GVT mode number

Fig. 12 Cross-orthogonality matrix of the baseline model (check mode shapes). 


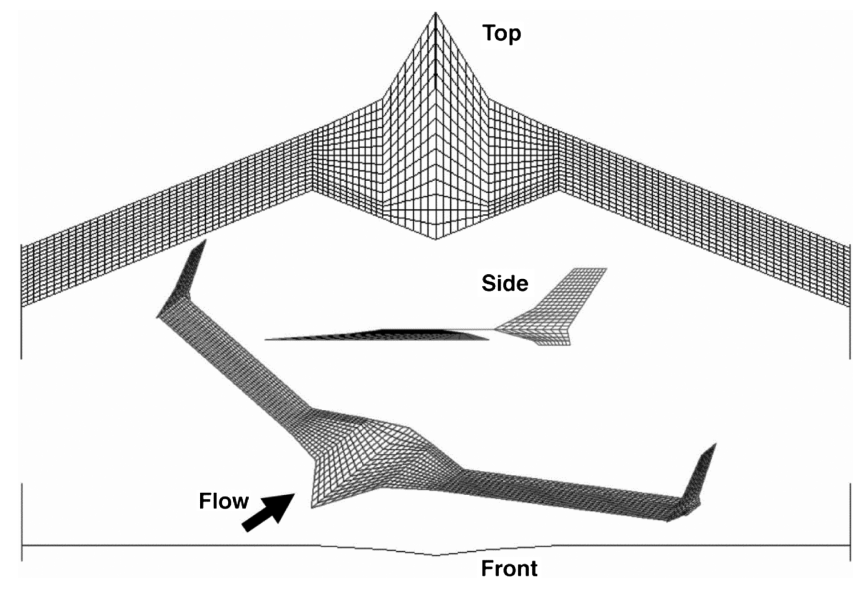

Fig. 13 Unsteady aerodynamic model of the X-56A aircraft.
12. Most of the off-diagonal terms of orthogonality and crossorthogonality matrices were less than $10 \%$; however, a few off-diagonal terms were still larger than $10 \%$. From these observations with Table 1 and Figs. 10-12, it was concluded that LMSW's baseline model still needs to be updated further to have an improvement in accuracy.

B. Identification of the Primary and Secondary Modes

One of the primary objectives of the X-56A aircraft is the flight demonstration of an active flutter suppression system. In this study, the primary and secondary modes for model tuning were identified through the use of modal participation factors obtained from a flutter analysis of the X-56A aircraft. An unsteady aerodynamic model for the flutter analysis using ZAERO code [29] is shown in Fig. 13. This aerodynamic model has 2196 surface elements over the center body, the wings, and the winglets. The matched flutter analyses were performed at four Mach numbers of 0.130, 0.160, 0.195, and 0.284. The speed versus damping $V-g$ and speed versus frequency $V-f$
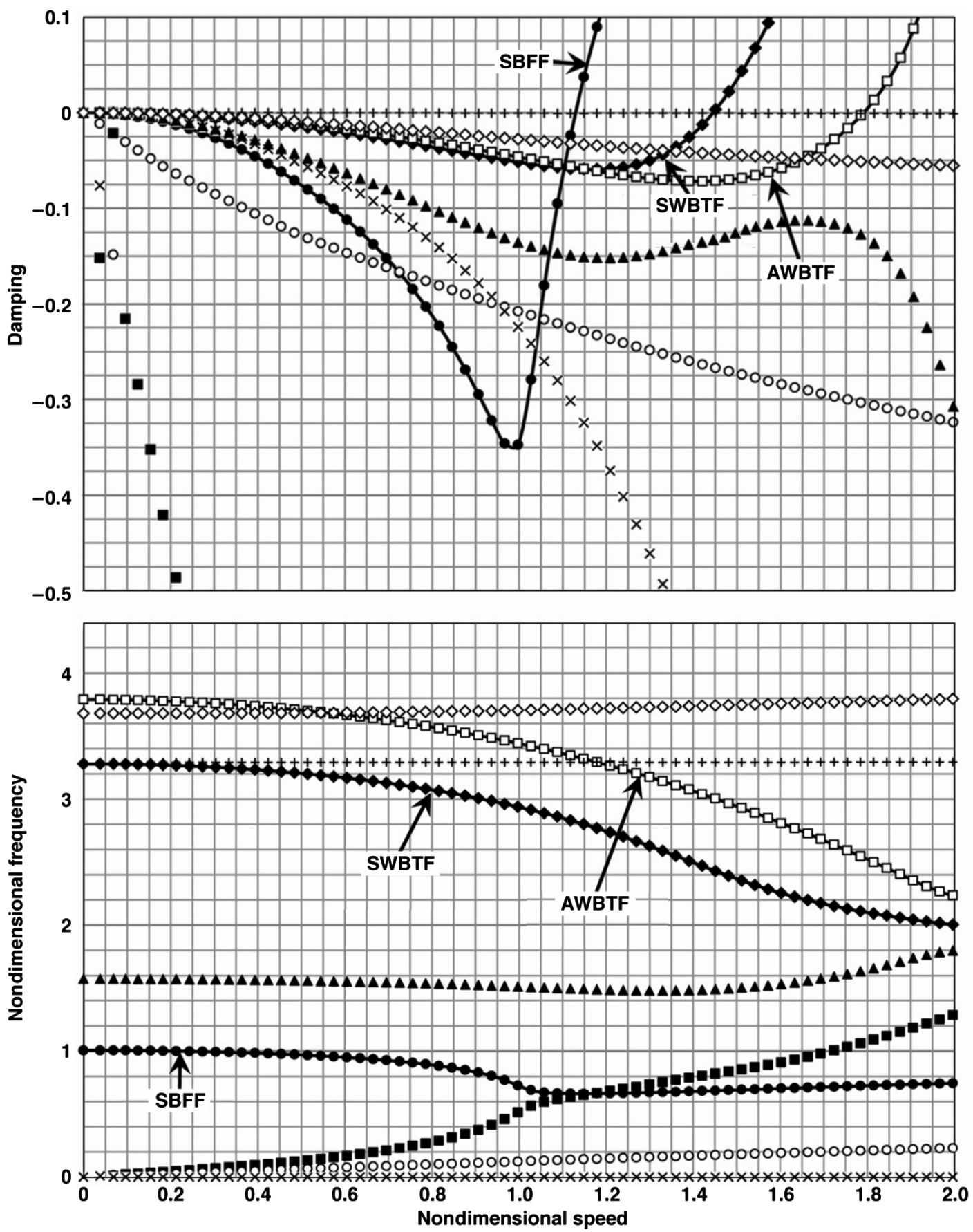

Fig. $14 V-g$ and $V-f$ curves of the baseline model with the EFEW at Mach 0.16. 
curves of the baseline model with the EFEW configuration at Mach number of 0.160 from the matched flutter analyses are given in Fig. 14. For comparison, the $V-g$ and $V-f$ curves of the final design model with the EFEW configuration are also presented in Fig. 15. The structural damping used for the flutter speed computations was $1 \%$. The flutter speeds and frequencies are summarized in Table 3 and Fig. 16. It should be noted in Fig. 16 that the first flutter mode is a symmetric body freedom flutter ( $\mathrm{SB} \overline{F F}$ ) (dashed line), the second flutter mode is a symmetric wing bending torsion flutter (SWBTF) (dashed single dot line), and the third flutter mode is an antisymmetric wing bending torsion flutter (AWBTF) (dashed double dot line).

Flutter mode shapes at Mach numbers of 0.130 and 0.284 are shown in Fig. 17. In Fig. 17, center body longitudinal motions together with the outboard wing bending motion can be observed in the first SBFF motion. These outboard wing bending motions are more of a divergence type of motion or wash in motion. On the other hand, small center body pitch and wing bending in addition to torsion motions are noticed in the first symmetric flutter mode. Small center body roll and outboard wing wash out bending and torsion motion can be seen in the case of the first antisymmetric flutter mode. An antisymmetric body freedom flutter mode is captured with a Mach number of 0.284 (open diamond marker in Fig. 16), and the corresponding mode shape is also given in Fig. 17.

Modal participation factors are summarized in Table 4 . In the case of the final design model, modal participation of mode numbers 7,8 , 9 , and 11 together with rigid body modes are $89.0-96.5 \%$ for all three Mach number cases as shown in Table $\underline{4}$. Therefore, these modes were the primary modes for the final design model. However, in the case of the baseline model, modal participation of the same modes was reduced to $84.7-93.0 \%$ for the SBFF mode, $76.3-89.5 \%$ for the symmetric flutter mode, and $33.6-68.3 \%$ for the antisymmetric
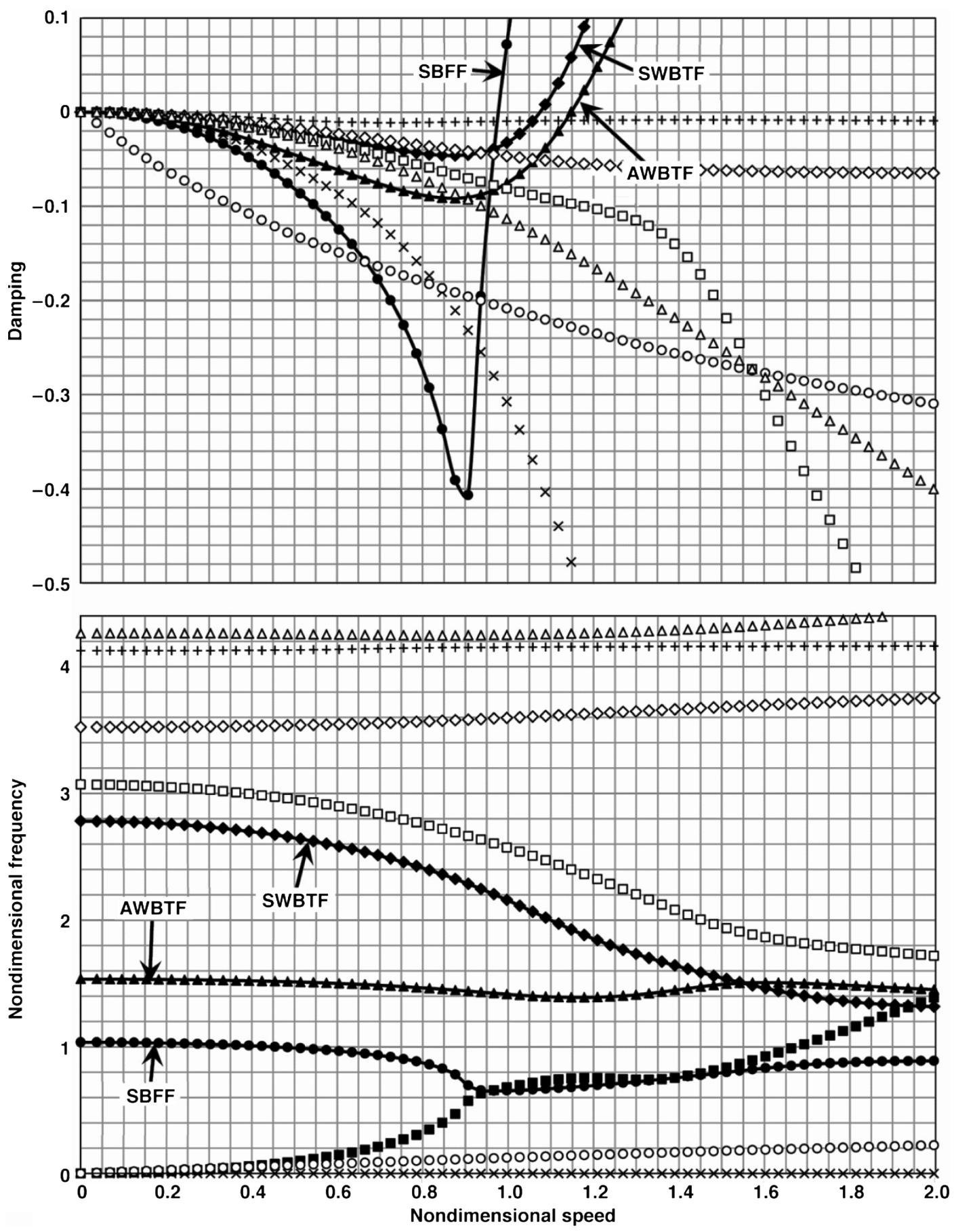

Fig. $15 V-g$ and $V-f$ curves of the final design model with the EFEW at Mach 0.16. 
Final design (non-validated)

Symmetric body freedom flutter

Symmetric wing bending torsion flutter

Anti-symmetric wing bending torsion flutter
Baseline (validated by LMSW)

O Symmetric body freedom flutter=

$\square$ Symmetric wing bending torsion flutter

$\diamond$ Anti-symmetric wing bending torsion flutter

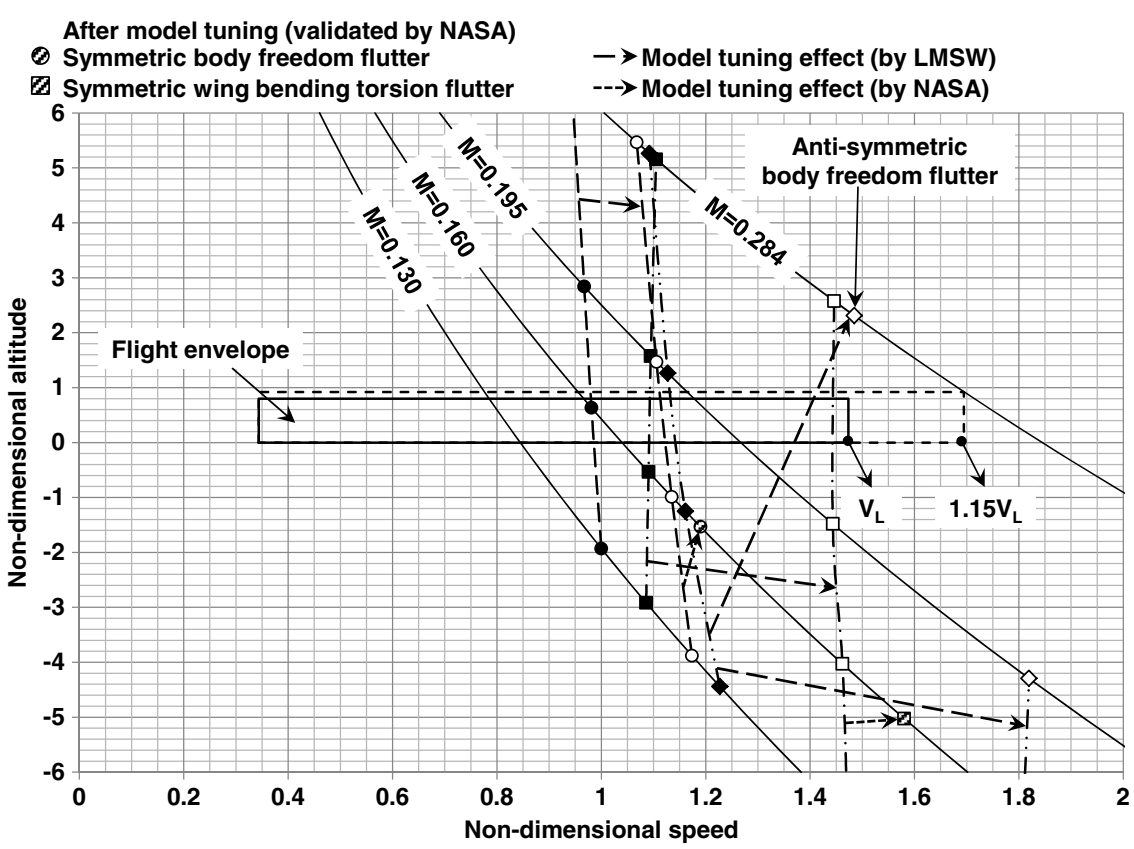

Fig. 16 Flutter boundaries and flight envelope under the EFEW configuration.

flutter mode. Therefore, mode numbers 12, 13, and 14 for the EFEW configuration and the same mode numbers for the FFFW configuration were added to the previous primary mode to have modal participation of 93.3-98.9\%. It should be noted that mode number 14, the symmetric main landing gear lateral mode, involves an average of 7.4 and $8.4 \%$ for the symmetric wing bending torsion flutter in the case of the EFEW, and for the antisymmetric wing bending torsion flutter in the case of the FFFW, respectively. Other than these two cases, this mode contributes less than $2.6 \%$ of the symmetric body freedom flutter mode.

\section{Structural Dynamic Model Tuning}

In general, the major objective of a GVT is the modal validation of the structural dynamic FE model. Based on the results of the test and analysis correlation, if these correlation results violate the military and NASA standards $[27,28]$, then the FE model needs to be adjusted to match the test data.

In this study, based on the data shown in Table 1 and Figs. 10-12, the baseline model developed by LMSW is further updated to have improved correlation with test data. The four model-tuning procedures performed in this study used the DOT algorithm to save

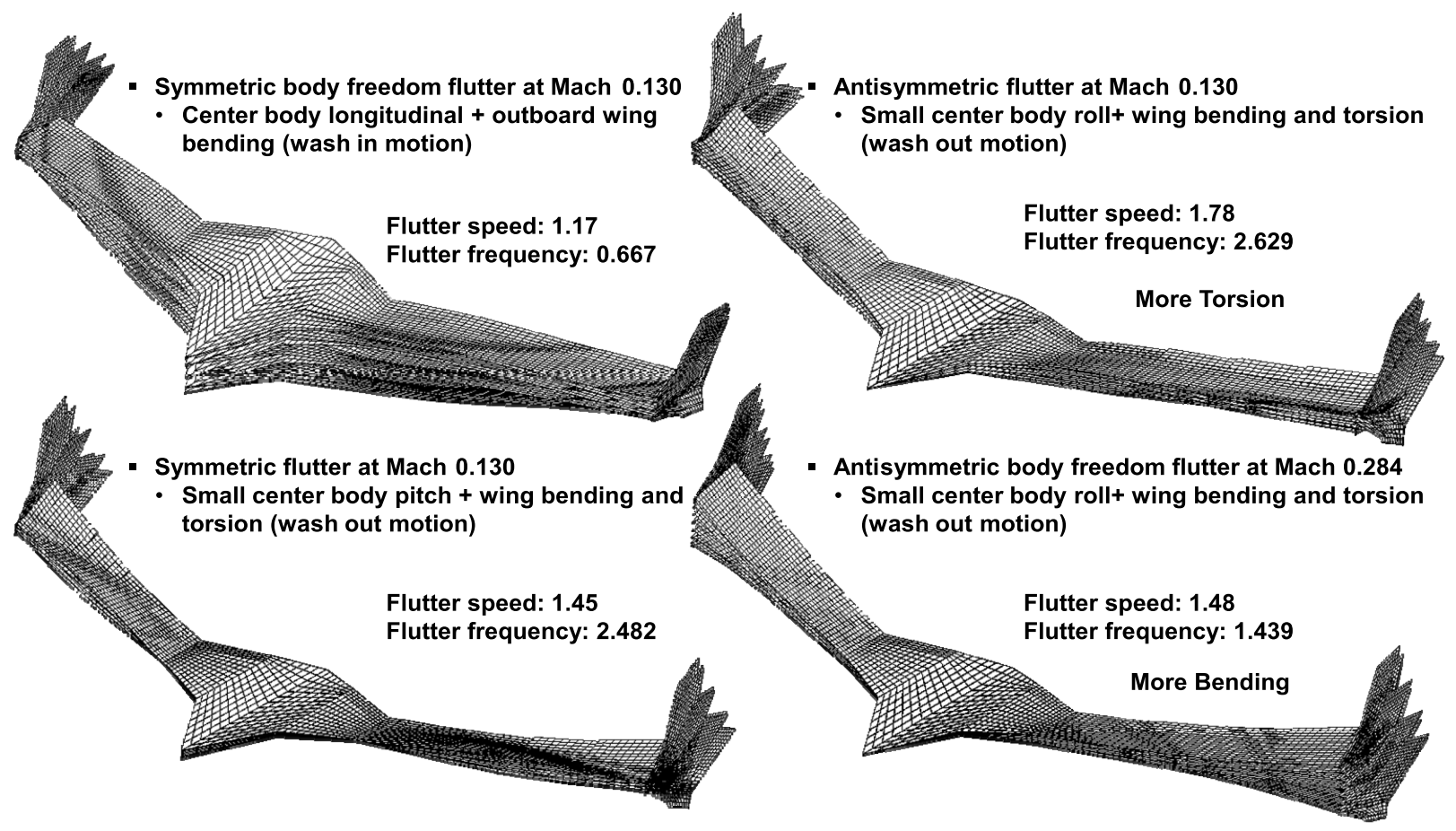

Fig. 17 First three flutter mode shapes of the X-56A baseline model. 
Table 3 Nondimensional flutter speeds and frequencies with EFEW at Mach 0.16

\begin{tabular}{|c|c|c|c|c|c|}
\hline \multirow[b]{2}{*}{ Flutter mode } & \multirow[b]{2}{*}{ Final design } & \multicolumn{2}{|r|}{ Baseline } & \multicolumn{2}{|c|}{ After fourth tuning } \\
\hline & & Speed & Difference, ${ }^{\mathrm{a}} \%$ & Speed & Difference, ${ }^{\mathrm{b}} \%$ \\
\hline \multicolumn{6}{|c|}{ Nondimensional flutter speed } \\
\hline First & 0.981 & 1.135 & 15.7 & 1.190 & 21.3 \\
\hline Second & 1.090 & 1.462 & 34.1 & 1.580 & 44.9 \\
\hline Third & 1.161 & 1.809 & 55.8 & 1.883 & 62.2 \\
\hline \multicolumn{6}{|c|}{ Nondimensional flutter frequency } \\
\hline First & 0.653 & 0.663 & 1.6 & 0.664 & 1.7 \\
\hline Second & 2.014 & 2.415 & 19.9 & 2.527 & 25.5 \\
\hline Third & 1.390 & 2.509 & 80.5 & 2.755 & 98.2 \\
\hline
\end{tabular}

${ }^{\mathrm{a}}$ Difference $=($ baseline-final design $) /$ final design.

${ }^{\mathrm{b}}$ Difference $=($ after tuning-final design $) /$ final design.

computation time. A summary of target objective functions to be improved for each model-tuning procedure are shown in Fig. 18.

\section{A. First Model-Tuning Procedure}

Frequency errors of the primary mode numbers 11 and 14 for the EFEW and FFFW configurations, with frequency errors of $-5.3,6.3$, -5.4 , and $7.4 \%$ in Table 1 , are selected as an objective function. The two modes, mode number 14 for the EFEW and FFFW configurations, are mainly related to the main landing gear. Therefore, one lumped mass value and nine sectional properties of the main landing gear, together with the corresponding Young's modulus $E$ and shear modulus $G$ were selected as design variables. It was assumed to have symmetric structural properties, and therefore design variable linking was used for these design variables. In this study, lumped mass values of the GVT sensor cables, $8 \mathrm{lb}$ in total, are also selected as design variables.

Differences in the total weights $(1+1), x$ c.g. locations $(1+1)$, all other frequencies $(13+10)$, the off-diagonal terms of symmetric orthogonality matrices $(21+21)$ in Eq. (13), and the off-diagonal terms of cross-orthogonality matrices $(42+42)$ in Eq. (14) were used as inequality constraints. Numbers in parenthesis designate that the first and second numbers are the number of inequality constraints obtained from the EFEW and FFFW configurations, respectively. Therefore, the total number of 12 design variables as well as 153 inequality constraints $(2+2+23+42+84)$ were used in this first optimization procedure. In this study, MAC values were not used for the constraint functions. Plus and minus $20 \%$ of the starting design variable values are selected as the upper and lower bounds for design variables (i.e., side constraints), respectively.

Continuous design variables were assumed, and model tuning was based on the DOT algorithm in the $\mathrm{O}^{3}$ tool. Updated frequency results and the MAC matrix are shown in Table 5 and Fig. 19, respectively. Orthogonality and cross-orthogonality matrices for the EFEW and FFFW configurations after the first model-tuning procedure are given in Figs. 20, 21. After the first model tuning, four frequencies related to the objective function improved from $-5.3,6.3,-5.4$, and $7.4 \%$ to $-3.5,5.6,-3.9$, and $6.7 \%$, respectively. Mode numbers 11 and 14 for the EFEW and FFFW configurations after the first model tuning still violate the military standard for primary modes (3\%). However, mode number 11 for these two weight configurations satisfied the NASA standard for primary modes $(5 \%)$.

In Fig. 20, small improvements for the off-diagonal terms of the orthogonality matrix were observed; 0.143 and 0.150 became 0.140 and 0.146 , respectively. On the other hand, -0.143 for the EFEW case became -0.144 , which was mainly because, when upper limit values of the constraint were prepared, these values were rounded up -0.143 to -0.144 to give some buffer value. This off-diagonal term was an active constraint, and therefore the optimizer tried to increase this constraint function during the first optimization procedure.

Big improvements in off-diagonal terms of the cross-orthogonality matrix were observed as shown in Fig. 21. In addition, a few offdiagonal terms were approaching the $10 \%$ target value. It should be noted that four off-diagonal terms violated military and NASA standards; $-24.4,13.3,-13.3$, and $-10.9 \%$, in Fig. 12, were less than
$10 \%$ target value after the first model tuning, and 9.2, 2.6, -11.0, and $-7.6 \%$, in Fig. 21.

It should be emphasized that the frequencies of the mode numbers 12 and 13 for the EFEW weight configuration were too close, as shown in Table 5, and the frequency difference was less than 0.001 (nondimensional frequency). As shown in Fig. 19, these two modes were not interchanged yet. Once mode interchange happens during a model-tuning procedure, then all the off-diagonal terms of the orthogonality and cross-orthogonality matrices will be discontinuous, and therefore the sensitivity matrices computed for finding the search direction and surface slope changes will be corrupted. To overcome this mode interchange problem, a special mode-tracking program based on diagonal and off-diagonal terms of the MAC matrix was developed and implemented into the current extended model-tuning tool. This program helped with the continuity of the performance indices.

\section{B. Second Model-Tuning Procedure}

For the second tuning procedure, mode numbers 12 and 14 of the cross-orthogonality matrix for the EFEW weight configuration were selected as the objective functions; specifically, the objective functions selected were off-diagonal terms 12-14 and 14-12. From Fig. 21, after the first model-tuning procedure, these off-diagonal terms resulted in an error of nearly 36 and 50\%, respectively, and needed to be improved further. It should be noted that four improved frequencies, mode numbers 11 and 14 for the two weight configurations, were switched to the constraint functions for this run. In this procedure, 20 design variables (eight from the last procedure, four from GVT sensor cable masses, and eight additional from main landing gear properties) were selected from a sensitivity analysis. This decision making is due to the fact that mode number 14 for both weight configurations is related directly to the main landing gear. In addition, upper and lower bounds for these design variables were kept at $\pm 20 \%$. All other cross-orthogonality matrix terms $(40+42)$ were used as constraint functions along with the remainder of the orthogonality matrix terms $(21+21)$, frequency terms $(15+12)$, total weight $(1+1), x$ c.g. locations $(1+1)$, and GVT sensor cable weight (1) for a total of 156 constraint functions. Like the previous run, no MAC constraints were used.

It should be noted here that mode number 12 of the EFEW weight configuration can be interchanged with mode number 13 during the second model-tuning procedure. Actually, the main reason why a special mode-tracking routine was developed in this study was due to

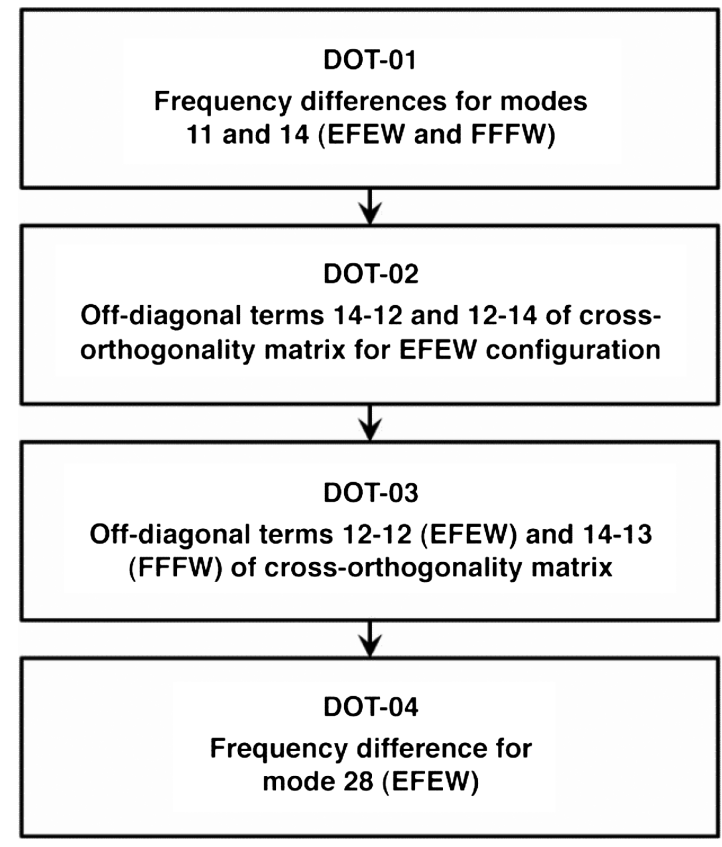

Fig. 18 Summary of objective functions. 
Table 4 Modal participation factors (percent) (first, second, and third flutter modes)

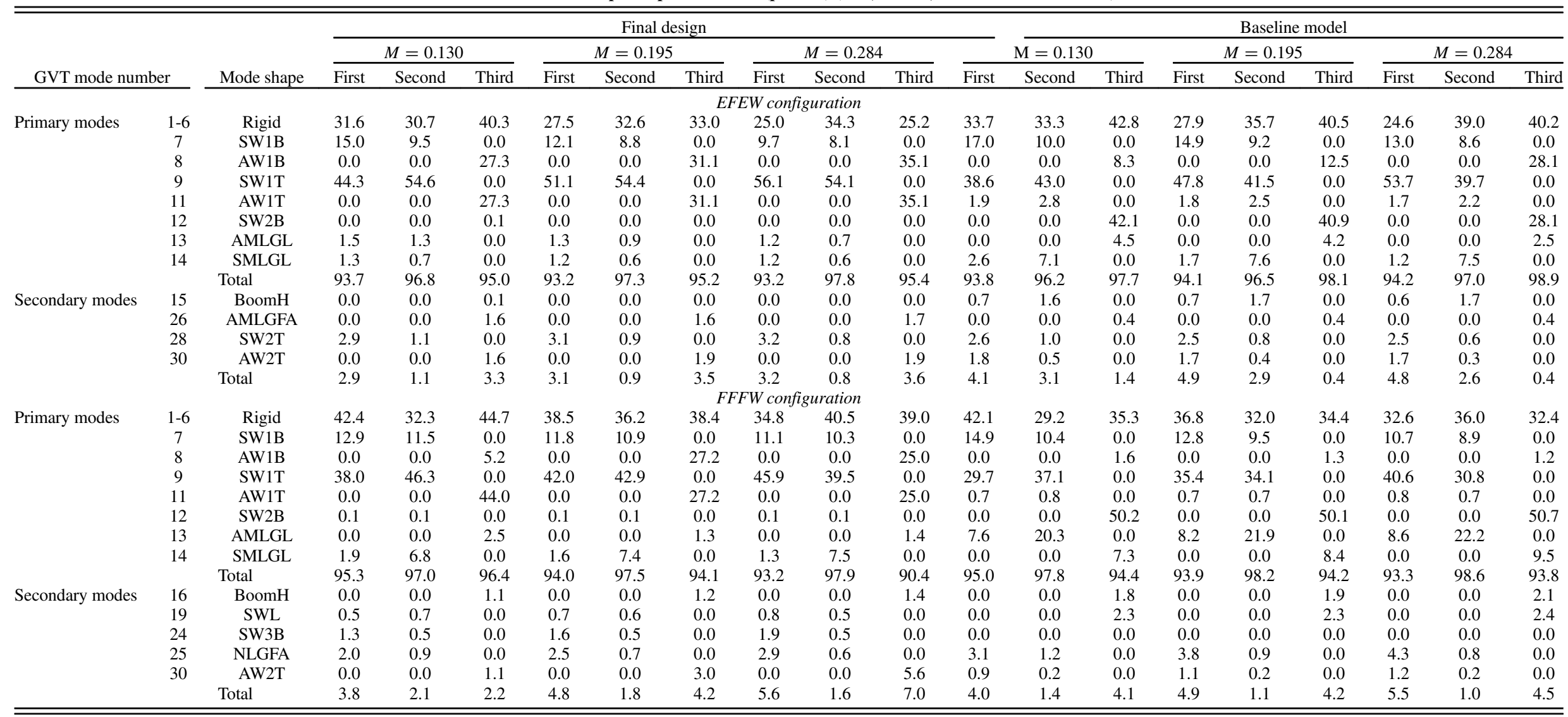




\begin{tabular}{|c|c|c|c|c|c|c|c|c|}
\hline \multicolumn{9}{|c|}{ EFEW configuration } \\
\hline & Mode shape & SW1B & AW1B & SW1T & AW1T & SW2B & AMLGL & SMLGL \\
\hline Mode shape & Mode number & $7^{*}$ & 8 & 9 & 11 & 12 & 13 & 14 \\
\hline SW1B & $7^{* *}$ & $\mathbf{0 . 9 9}$ & 0.00 & 0.00 & 0.00 & 0.08 & 0.00 & 0.01 \\
\hline AW1B & 8 & 0.00 & $\mathbf{1 . 0 0}$ & 0.00 & 0.00 & 0.00 & 0.09 & 0.00 \\
\hline SW1T & 9 & 0.01 & 0.00 & $\mathbf{0 . 9 2}$ & 0.00 & 0.00 & 0.00 & 0.01 \\
\hline AW1T & 11 & 0.00 & 0.01 & 0.00 & $\mathbf{0 . 9 8}$ & 0.01 & 0.17 & 0.00 \\
\hline SW2B & 12 & 0.09 & 0.00 & 0.07 & 0.01 & $\mathbf{0 . 9 8}$ & 0.00 & 0.30 \\
\hline AMLGL & 13 & 0.00 & 0.06 & 0.00 & 0.41 & 0.00 & $\mathbf{0 . 9 7}$ & 0.02 \\
\hline SMLGL & 14 & 0.05 & 0.00 & 0.06 & 0.00 & 0.92 & 0.03 & $\mathbf{0 . 5 5}$ \\
\hline \hline \multicolumn{7}{|c|}{ FFFW configuration } \\
\hline Mode shape & Mode number & $7 *$ & 8 & 9 & 11 & 12 & 13 & 14 \\
\hline SW1B & $7 * *$ & $\mathbf{0 . 9 9}$ & 0.00 & 0.02 & 0.00 & 0.08 & 0.00 & 0.01 \\
\hline AW1B & 8 & 0.00 & $\mathbf{1 . 0 0}$ & 0.00 & 0.02 & 0.00 & 0.07 & 0.00 \\
\hline SW1T & 9 & 0.04 & 0.00 & $\mathbf{0 . 9 5}$ & 0.00 & 0.00 & 0.00 & 0.02 \\
\hline AW1T & 11 & 0.00 & 0.04 & 0.00 & $\mathbf{0 . 9 3}$ & 0.01 & 0.10 & 0.01 \\
\hline SW2B & 12 & 0.07 & 0.00 & 0.04 & 0.00 & $\mathbf{0 . 9 9}$ & 0.00 & 0.15 \\
\hline AMLGL & 13 & 0.00 & 0.04 & 0.00 & 0.33 & 0.01 & $\mathbf{0 . 9 7}$ & 0.01 \\
\hline SMLGL & 14 & 0.03 & 0.00 & 0.03 & 0.00 & 0.85 & 0.02 & $\mathbf{0 . 3 8}$ \\
\hline
\end{tabular}

*: Nastran mode number

**: GVT mode number

Fig. 19 MAC matrix after the first model-tuning procedure (DOT-01).

this mode interchange. The MAC matrix after the second modeltuning procedure is given in Fig. 22.

After several optimization iterations, it turned out that the frequency errors of mode number 14 (SMLGL) and mode number 28 (AMLGFA) for the EFEW configuration, off-diagonal term 12-13 (AMLGL SW2B) of the orthogonality matrix for the EFEW configuration, and finally off-diagonal term 14-13 (AMLGL-SMLGL) of the cross-orthogonality matrix became active constraints, and no improvements were observed. Therefore, the constraint limit values were relaxed to give more allowance for improvement, 6 and $20 \%$ upper limit for frequency errors, $15 \%$ for off-diagonal terms $12-13$ and $14-13$ of the orthogonality and cross-orthogonality matrices.

Model tuning was performed using the DOT algorithm. Frequency results for this second tuning procedure are also shown in Table 5. Orthogonality and cross-orthogonality matrices for the EFE $\bar{W}$ and FFFW configurations are presented in Figs. 23, 24. For both configurations, frequency errors did not change significantly. Even with relaxed upper limit values, frequency errors for modes 14 and 28 did not change at all.
Results for the orthogonality matrices for both weight configurations after the second procedure are shown in Fig. 23. Note that, for this procedure, all of the off-diagonal terms for the orthogonality matrices were selected as constraints, not objective functions. Similarly, orthogonality matrices for both weight configurations were unchanged during the second optimization run. Off-diagonal term 12-13 was an active constraint, and this term did not become better or worse even with relaxed upper limit value.

Results for the off-diagonal terms of the cross-orthogonality matrix after the second tuning procedure are shown in Fig. 24. In comparison to the first tuning procedure results in Fig. 21, some off-diagonal terms, mostly constraints, slightly improved for both configurations. However, the objective functions for this procedure were off-diagonal terms 12-14 and 14-12 for the EFEW configuration and have improved negligibly from -0.497 to -0.495 and from -0.358 to -0.357 .

\section{Third Model-Tuning Procedure}

Based on the results from the second model-tuning procedure, there was very minimal improvement in the cross-orthogonality

\begin{tabular}{|c|c|c|c|c|c|c|c|c|}
\hline \multicolumn{9}{|c|}{ EFEW configuration } \\
\hline & Mode shape & SW1B & AW1B & SW1T & AW1T & SW2B & AMLGL & SMLGL \\
\hline Mode shape & Mode number & $7^{* *}$ & 8 & 9 & 11 & 12 & 13 & 14 \\
\hline SW1B & $7^{* *}$ & $\mathbf{1 . 0 0 0}$ & -0.021 & -0.053 & -0.011 & 0.026 & 0.024 & -0.033 \\
\hline AW1B & 8 & -0.021 & $\mathbf{1 . 0 0 0}$ & 0.012 & 0.002 & 0.004 & 0.039 & 0.002 \\
\hline SW1T & 9 & -0.053 & 0.012 & $\mathbf{1 . 0 0 0}$ & 0.004 & 0.035 & -0.007 & -0.025 \\
\hline AW1T & 11 & -0.011 & 0.002 & 0.004 & $\mathbf{1 . 0 0 0}$ & 0.021 & -0.091 & 0.003 \\
\hline SW2B & 12 & 0.026 & 0.004 & 0.035 & 0.021 & $\mathbf{1 . 0 0 0}$ & -0.144 & 0.140 \\
\hline AMLGL & 13 & 0.024 & 0.039 & -0.007 & -0.091 & -0.144 & $\mathbf{1 . 0 0 0}$ & 0.005 \\
\hline SMLGL & 14 & -0.033 & 0.002 & -0.025 & 0.003 & 0.140 & 0.005 & $\mathbf{1 . 0 0 0}$ \\
\hline \hline \multicolumn{7}{|c|}{ FFFW configuration } & & \\
\hline \\
\hline Mode shape & Mode number & $7 * *$ & 8 & 9 & 11 & 12 & 13 & 14 \\
\hline SW1B & $7 * *$ & $\mathbf{1 . 0 0 0}$ & 0.013 & -0.047 & 0.014 & 0.019 & 0.008 & -0.065 \\
\hline AW1B & 8 & 0.013 & $\mathbf{1 . 0 0 0}$ & -0.010 & 0.013 & -0.005 & -0.062 & -0.011 \\
\hline SW1T & 9 & -0.047 & -0.010 & $\mathbf{1 . 0 0 0}$ & -0.019 & 0.007 & -0.013 & -0.026 \\
\hline AW1T & 11 & 0.014 & 0.013 & -0.019 & $\mathbf{1 . 0 0 0}$ & -0.026 & 0.092 & 0.017 \\
\hline SW2B & 12 & 0.019 & -0.005 & 0.007 & -0.026 & $\mathbf{1 . 0 0 0}$ & 0.003 & 0.146 \\
\hline AMLGL & 13 & 0.008 & -0.062 & -0.013 & 0.092 & 0.003 & $\mathbf{1 . 0 0 0}$ & -0.076 \\
\hline SMLGL & 14 & -0.065 & -0.011 & -0.026 & 0.017 & 0.146 & -0.076 & $\mathbf{1 . 0 0 0}$ \\
\hline
\end{tabular}

**: GVT mode number

Fig. 20 Orthogonality matrix after the first model-tuning procedure (DOT-01). 


\begin{tabular}{|c|c|c|c|c|c|c|c|c|}
\hline \multicolumn{9}{|c|}{ EFEW configuration } \\
\hline & Mode shape & SW1B & AW1B & SW1T & AW1T & SW2B & AMLGL & SMLGL \\
\hline Mode shape & Mode number & 7* & 8 & 9 & 11 & 12 & 13 & 14 \\
\hline SW1B & $7 * *$ & 1.006 & 0.015 & 0.034 & 0.007 & -0.022 & -0.015 & -0.064 \\
\hline AW1B & 8 & -0.006 & -1.018 & -0.004 & 0.008 & 0.000 & -0.030 & 0.003 \\
\hline SW1T & 9 & -0.028 & -0.007 & -1.038 & -0.020 & 0.106 & 0.021 & -0.021 \\
\hline AW1T & 11 & 0.000 & -0.011 & -0.003 & -1.032 & 0.001 & -0.064 & -0.002 \\
\hline SW2B & 12 & 0.000 & -0.004 & -0.126 & -0.019 & -0.894 & 0.092 & -0.358 \\
\hline AMLGL & 13 & 0.009 & -0.015 & -0.016 & 0.180 & 0.026 & -0.983 & -0.002 \\
\hline SMLGL & 14 & 0.010 & -0.003 & -0.071 & 0.006 & $\overline{-0.497}$ & -0.009 & 0.759 \\
\hline \multicolumn{9}{|c|}{ FFFW configuration } \\
\hline & Mode shape & SW1B & AW1B & SW1T & AW1T & SW2B & AMLGL & SMLGL \\
\hline Mode shape & Mode number & 7* & 8 & 9 & 11 & 12 & 13 & 14 \\
\hline SW1B & $7 * *$ & -1.086 & -0.008 & -0.037 & 0.012 & 0.033 & 0.012 & 0.061 \\
\hline AW1B & 8 & -0.003 & -1.109 & -0.008 & 0.011 & -0.004 & -0.059 & 0.011 \\
\hline SW1T & 9 & 0.026 & 0.004 & 1.146 & -0.016 & $\underline{-0.110}$ & -0.007 & 0.016 \\
\hline AW1T & 11 & 0.019 & 0.001 & -0.032 & 1.082 & 0.014 & -0.076 & -0.009 \\
\hline SW2B & 12 & 0.009 & 0.003 & 0.112 & -0.005 & 1.046 & -0.053 & 0.205 \\
\hline AMLGL & 13 & 0.003 & 0.010 & 0.002 & 0.180 & 0.072 & 1.036 & 0.002 \\
\hline SMLGL & 14 & 0.011 & 0.011 & 0.038 & 0.014 & 0.340 & -0.113 & -0.843 \\
\hline
\end{tabular}

*: Nastran mode number

**: GVT mode number

Fig. 21 Cross-orthogonality matrix after the first model-tuning procedure (DOT-01).

matrix as shown in Fig. 24, when compared to the first model-tuning run in Fig. 21. The objective functions and corresponding design variables during the previous model-tuning procedure were reviewed before conducting this third model-tuning procedure. By looking at some of the previous designs, there was a possibility that a better design configuration existed. Just because the output of the previous procedure resulted in miniscule improvement does not imply that the $\mathrm{O}^{3}$ tool program did not find a better optimization configuration in one of its iterations. From the previous optimization history (POH), a better starting configuration was selected. This design configuration was not selected by the DOT because some of the performance indices had violated constraint functions, which were actually acceptable violations. The results from this selected configuration are displayed in Table 6 and Figs. $25-27$ for frequencies, the MAC matrix, the mass orthogonality matrix, and the cross-orthogonality matrix, respectively.
When comparing frequency results in Tables 5 and 6 , it can be seen that there was a mode interchange between modes 12 and 13 for both weight configurations as the frequencies were switched. As shown in Tables $\underline{5}$ and $\underline{6}$, frequencies of mode number 14 for the EFEW and FFFW configurations after DOT-02 in Table 5, 5.6 and 6.7\%, were all less than $5 \%$ (even smaller than 3\%) in Table 6 (under POH). Also, notice that the frequency for mode 28 of the EFEW weight configuration reached the constraint value limit of 20\%. In Fig. 25, the MAC values for mode 14 of the EFEW and FFFW configurations based on the selected POH was 0.97 and 0.61 , respectively; these MAC values are far better than the 0.55 and 0.39 from DOT-02 as shown in Fig. 22. In addition, the mass orthogonality matrices for both weight configurations improved as well, as shown in Fig. 26; however, for the EFEW, off-diagonal term 12-13 increased when compared to the second model-tuning results, whereas off-diagonal term 12-14 improved to 0.107 when compared to 0.140 in Fig. 23 . For

\begin{tabular}{|c|c|c|c|c|c|c|c|c|}
\hline \multicolumn{9}{|c|}{ EFEW configuration } \\
\hline & Mode shape & SW1B & AW1B & SW1T & AW1T & SW2B & AMLGL & SMLGL \\
\hline Mode shape & Mode number & $7^{*}$ & 8 & 9 & 11 & 12 & 13 & 14 \\
\hline SW1B & $7^{* *}$ & $\mathbf{0 . 9 9}$ & 0.00 & 0.00 & 0.00 & 0.08 & 0.01 & 0.01 \\
\hline AW1B & 8 & 0.00 & $\mathbf{1 . 0 0}$ & 0.00 & 0.00 & 0.00 & 0.08 & 0.00 \\
\hline SW1T & 9 & 0.01 & 0.00 & $\mathbf{0 . 9 2}$ & 0.00 & 0.00 & 0.00 & 0.01 \\
\hline AW1T & 11 & 0.00 & 0.01 & 0.00 & $\mathbf{0 . 9 8}$ & 0.02 & 0.16 & 0.00 \\
\hline SW2B & 12 & 0.09 & 0.00 & 0.07 & 0.01 & $\mathbf{0 . 9 8}$ & 0.01 & 0.30 \\
\hline AMLGL & 13 & 0.00 & 0.06 & 0.00 & 0.41 & 0.00 & $\mathbf{0 . 9 5}$ & 0.02 \\
\hline SMLGL & 14 & 0.05 & 0.00 & 0.06 & 0.00 & 0.91 & 0.07 & $\mathbf{0 . 5 5}$ \\
\hline \hline \multicolumn{7}{|c|}{ FFFW configuration } & & \\
\hline Mode shape & Mode number & SW1B & AW1B & SW1T & AW1T & SW2B & AMLGL & SMLGL \\
\hline SW1B & $7 * *$ & 9 & 11 & 12 & 13 & 14 \\
\hline AW1B & 8 & $\mathbf{0 . 9 9}$ & 0.00 & 0.02 & 0.00 & 0.08 & 0.00 & 0.01 \\
\hline SW1T & 9 & 0.00 & $\mathbf{1 . 0 0}$ & 0.00 & 0.02 & 0.00 & 0.07 & 0.00 \\
\hline AW1T & 11 & 0.04 & 0.00 & $\mathbf{0 . 9 5}$ & 0.00 & 0.00 & 0.00 & 0.02 \\
\hline SW2B & 12 & 0.07 & 0.00 & 0.04 & 0.00 & $\mathbf{0 . 9 9}$ & 0.00 & 0.15 \\
\hline AMLGL & 13 & 0.00 & 0.04 & 0.00 & 0.33 & 0.01 & $\mathbf{0 . 9 7}$ & 0.01 \\
\hline SMLGL & 14 & 0.03 & 0.00 & 0.03 & 0.00 & 0.85 & 0.02 & $\mathbf{0 . 3 9}$ \\
\hline
\end{tabular}

*: Nastran mode number

**: GVT mode number

Fig. 22 MAC matrix after the second model-tuning procedure (DOT-02). 


\begin{tabular}{|c|c|c|c|c|c|c|c|c|}
\hline \multicolumn{9}{|c|}{ EFEW configuration } \\
\hline & Mode shape & SW1B & AW1B & SW1T & AW1T & SW2B & AMLGL & SMLGL \\
\hline Mode shape & Mode number & $7^{* *}$ & 8 & 9 & 11 & 12 & 13 & 14 \\
\hline SW1B & $7^{* *}$ & $\mathbf{1 . 0 0 0}$ & -0.021 & -0.053 & -0.011 & 0.026 & 0.024 & -0.033 \\
\hline AW1B & 8 & -0.021 & $\mathbf{1 . 0 0 0}$ & 0.012 & 0.002 & 0.004 & 0.039 & 0.002 \\
\hline SW1T & 9 & -0.053 & 0.012 & $\mathbf{1 . 0 0 0}$ & 0.004 & 0.035 & -0.007 & -0.025 \\
\hline AW1T & 11 & -0.011 & 0.002 & 0.004 & $\mathbf{1 . 0 0 0}$ & 0.021 & -0.091 & 0.003 \\
\hline SW2B & 12 & 0.026 & 0.004 & 0.035 & 0.021 & $\mathbf{1 . 0 0 0}$ & -0.144 & 0.140 \\
\hline AMLGL & 13 & 0.024 & 0.039 & -0.007 & -0.091 & -0.144 & $\mathbf{1 . 0 0 0}$ & 0.005 \\
\hline SMLGL & 14 & -0.033 & 0.002 & -0.025 & 0.003 & 0.140 & 0.005 & $\mathbf{1 . 0 0 0}$ \\
\hline \multicolumn{7}{|c|}{ FFFW configuration } \\
\hline Mode shape & Mode number & $7^{* *}$ & AW1B & SW1T & AW1T & SW2B & AMLGL & SMLGL \\
\hline SW1B & $7 * *$ & $\mathbf{1 . 0 0 0}$ & 0.013 & -0.047 & 0.014 & 0.019 & 0.008 & -0.065 \\
\hline AW1B & 8 & 0.013 & $\mathbf{1 . 0 0 0}$ & -0.010 & 0.013 & -0.005 & -0.062 & -0.011 \\
\hline SW1T & 9 & -0.047 & -0.010 & $\mathbf{1 . 0 0 0}$ & -0.019 & 0.007 & -0.013 & -0.026 \\
\hline AW1T & 11 & 0.014 & 0.013 & -0.019 & $\mathbf{1 . 0 0 0}$ & -0.026 & 0.092 & 0.017 \\
\hline SW2B & 12 & 0.019 & -0.005 & 0.007 & -0.026 & $\mathbf{1 . 0 0 0}$ & 0.003 & 0.146 \\
\hline AMLGL & 13 & 0.008 & -0.062 & -0.013 & 0.092 & 0.003 & $\mathbf{1 . 0 0 0}$ & -0.076 \\
\hline SMLGL & 14 & -0.065 & -0.011 & -0.026 & 0.017 & 0.146 & -0.076 & $\mathbf{1 . 0 0 0}$ \\
\hline
\end{tabular}

**: GVT mode number

Fig. 23 Orthogonality matrix after the second model-tuning procedure (DOT-02).

the FFFW, off-diagonal term 12-14 also significantly improved from 0.146 to 0.118 .

Furthermore, Fig. 27 displays a strong improvement in the crossorthogonality matrices when compared to Fig. 24 after the second model-tuning procedure. After the first model-tuning procedure in Fig. 21, off-diagonal terms 12-13 and 13-12 for the EFEW configuration were constrained to be a maximum value of $10 \%$. After the second model-tuning procedure, in Fig. 24, these two offdiagonal terms satisfied the $10 \%$ requirement. Although these two off-diagonal terms in Fig. 27 violated the $10 \%$ requirement; however, compared to Fig. 24 results, the cross-orthogonality matrix in Fig. 27 had a more improved configuration when compared to the results in Fig. 24. Significant improvements were observed for the EFEW offdiagonal terms $12-14$ and $14-12$, which went from -0.357 to -0.139 and -0.495 to 0.000 , respectively. Likewise, the FFFW off-diagonal terms 12-14 and 14-12 (14-13 due to mode interchange) improved magnitudewise from 0.205 to 0.162 and from 0.340 to 0.286 . On the other hand, some mode shape off-diagonal terms did not improve; most significant was the EFEW off-diagonal term 12-13 (12-12 due to mode interchange), which was 0.030 after the second modeltuning procedure but was 0.238 based on $\mathrm{POH}$. Overall, this $\mathrm{POH}$ configuration is much better than the results after the second modeltuning procedure. By selecting an improved optimization configuration based on the $\mathrm{POH}$ as the new starting point for this run, the optimization results for this third model-tuning procedure can be improved significantly.

Based on the cross-orthogonality matrix from the $\mathrm{POH}$ in Fig. 27, off-diagonal terms 12-12 (SW2B-AMLGL) of the EFEW configuration and 14-13 (SMLGL-SW2B) of the FFFW configuration were selected as the objective functions for this third modeltuning procedure. The same design variables were used as before, but with the addition of four main landing gear design variables, for a total of 24 design variables. Constraint functions consisted of all other cross-orthogonality matrix terms $(41+41)$, mass orthogonality matrix terms $(21+21)$, frequency terms $(15+12)$, total weight $(1+1), x$ c.g. locations $(1+1)$, and GVT sensor cable

\begin{tabular}{|c|c|c|c|c|c|c|c|c|}
\hline \multicolumn{9}{|c|}{ EFEW configuration } \\
\hline & Mode shape & SW1B & AW1B & SW1T & AW1T & SW2B & AMLGL & SMLGL \\
\hline Mode shape & Mode number & $7^{*}$ & 8 & 9 & 11 & 12 & 13 & 14 \\
\hline SW1B & $7^{* *}$ & $\mathbf{- 1 . 0 0 6}$ & 0.015 & 0.034 & 0.007 & -0.022 & -0.017 & -0.064 \\
\hline AW1B & 8 & 0.006 & $\mathbf{- 1 . 0 1 8}$ & -0.004 & 0.008 & 0.002 & -0.030 & 0.003 \\
\hline SW1T & 9 & 0.028 & -0.007 & $\mathbf{- 1 . 0 3 8}$ & -0.020 & 0.104 & 0.028 & -0.021 \\
\hline AW1T & 11 & 0.000 & -0.011 & -0.003 & $\mathbf{- 1 . 0 3 2}$ & 0.005 & -0.064 & -0.002 \\
\hline SW2B & 12 & 0.000 & -0.004 & -0.126 & -0.019 & $\mathbf{- 0 . 8 9 8}$ & 0.030 & -0.357 \\
\hline AMLGL & 13 & -0.009 & -0.015 & -0.016 & 0.180 & 0.094 & $\mathbf{- 0 . 9 7 9}$ & -0.002 \\
\hline SMLGL & 14 & -0.010 & -0.003 & -0.071 & 0.006 & -0.495 & -0.044 & $\mathbf{0 . 7 5 9}$ \\
\hline \hline \multicolumn{7}{|c|}{ FFFW configuration } \\
\hline Mode shape & Mode number & SW1B & AW1B & SW1T & AW1T & SW2B & AMLGL & SMLGL \\
\hline SW1B & $7 * *$ & 9 & 9 & 11 & 12 & 13 & 14 \\
\hline AW1B & 8 & $\mathbf{1 . 0 8 6}$ & -0.008 & 0.037 & 0.012 & 0.033 & 0.012 & 0.061 \\
\hline SW1T & 9 & -0.026 & 0.004 & $-\mathbf{1 . 1 4 6}$ & -0.016 & -0.110 & -0.007 & 0.016 \\
\hline AW1T & 11 & -0.019 & 0.001 & 0.032 & $\mathbf{1 . 0 8 2}$ & 0.014 & $\underline{-0.076}$ & -0.009 \\
\hline SW2B & 12 & -0.009 & 0.003 & -0.112 & -0.005 & $\mathbf{1 . 0 4 6}$ & -0.053 & 0.205 \\
\hline AMLGL & 13 & -0.003 & 0.010 & -0.002 & 0.180 & 0.072 & $\mathbf{1 . 0 3 6}$ & 0.002 \\
\hline SMLGL & 14 & -0.011 & 0.011 & -0.038 & 0.014 & 0.340 & -0.113 & $\mathbf{- 0 . 8 4 3}$ \\
\hline
\end{tabular}

*: Nastran mode number

**: GVT mode number

Fig. 24 Cross-orthogonality matrix after the second model-tuning procedure (DOT-02). 
Table 5 Flexible modes after the first and second model-tuning procedures

\begin{tabular}{|c|c|c|c|c|c|c|c|c|}
\hline & & \multicolumn{6}{|c|}{ Nastran results } & \multirow[b]{3}{*}{ Target error, $\%$} \\
\hline \multicolumn{2}{|c|}{ GVT data } & \multicolumn{3}{|c|}{ DOT-01 } & \multicolumn{3}{|c|}{ DOT-02 } & \\
\hline Mode number & Mode shape & Mode number & Frequency & Error, \% & Mode number & Frequency & Error, \% & \\
\hline \multicolumn{9}{|c|}{ EFEW configuration } \\
\hline 7 & SW1B & 7 & 1.086 & 1.7 & 7 & 1.086 & 1.7 & 5 \\
\hline 8 & AW1B & 8 & 1.535 & -0.5 & 8 & 1.535 & -0.5 & 5 \\
\hline 9 & SW1T & 9 & 3.193 & -0.9 & 9 & 3.193 & -0.9 & 5 \\
\hline 11 & AW1T & 11 & 3.703 & -3.5 & 11 & 3.703 & -3.5 & 5 \\
\hline 12 & SW2B & 12 & 4.553 & 2.5 & 12 & 4.553 & 2.5 & 5 \\
\hline 13 & AMLGL & 13 & 4.554 & 2.0 & 13 & 4.554 & 2.0 & 5 \\
\hline 14 & SMLGL & 14 & 4.927 & 5.6 & 14 & 4.927 & 5.6 & 6 \\
\hline 15 & BoomH & 15 & 5.223 & -0.9 & 15 & 5.223 & -0.9 & 10 \\
\hline 18 & AW2B & 18 & 6.064 & 0.6 & 18 & 6.065 & 0.6 & 10 \\
\hline 19 & SWL & 19 & 6.197 & -1.1 & 19 & 6.197 & -1.1 & 10 \\
\hline 25 & SW3B & 25 & 9.413 & 0.7 & 25 & 9.414 & 0.7 & 10 \\
\hline 26 & AW3B & 27 & 11.042 & 4.2 & 27 & 11.042 & 4.2 & 10 \\
\hline 28 & AMLGFA & 26 & 10.009 & -16.1 & 26 & 10.009 & -16.1 & 20 \\
\hline 30 & AW2T & 30 & 12.894 & 3.9 & 30 & 12.894 & 3.9 & 10 \\
\hline \multicolumn{2}{|c|}{ Total weight } & \multicolumn{2}{|c|}{366.0} & -0.18 & \multicolumn{2}{|c|}{366.0} & -0.18 & 5 \\
\hline \multicolumn{2}{|c|}{$x$ c.g. location } & \multicolumn{2}{|c|}{164.7} & -0.16 & \multicolumn{2}{|c|}{164.7} & -0.16 & 5 \\
\hline \multicolumn{2}{|c|}{$y$ c.g. location } & \multicolumn{2}{|c|}{0.3} & -413 & \multicolumn{2}{|c|}{0.3} & -413 & \\
\hline \multicolumn{2}{|c|}{$z$ c.g. location } & 101. & & -- & 101. & & -- & -- \\
\hline \multicolumn{9}{|c|}{ FFFW configuration } \\
\hline 7 & SW1B & 7 & 0.997 & -0.3 & 7 & 0.997 & -0.3 & 5 \\
\hline 8 & AW1B & 8 & 1.394 & -1.2 & 8 & 1.394 & -1.2 & 5 \\
\hline 9 & SW1T & 9 & 2.935 & -0.1 & 9 & 2.935 & -0.1 & 5 \\
\hline 11 & AW1T & 11 & 3.509 & -3.9 & 11 & 3.510 & -3.9 & 5 \\
\hline 12 & SW2B & 12 & 4.336 & -0.2 & 12 & 4.336 & -0.2 & 5 \\
\hline 13 & AMLGL & 13 & 4.446 & 0.9 & 13 & 4.446 & 0.9 & 5 \\
\hline 14 & SMLGL & 14 & 4.909 & 6.7 & 14 & 4.909 & 6.7 & 6.7 \\
\hline 16 & BoomH & 16 & 5.217 & -1.1 & 16 & 5.217 & -1.1 & 10 \\
\hline 19 & SWL & 19 & 6.023 & -2.0 & 19 & 6.023 & -2.0 & 10 \\
\hline 24 & SW3B & 24 & 8.674 & 0.2 & 24 & 8.674 & 0.2 & 10 \\
\hline 25 & NLGFA & 25 & 9.186 & 0.6 & 25 & 9.186 & 0.6 & 10 \\
\hline 28 & AW2T & 30 & 11.776 & 2.0 & 30 & 11.776 & 2.0 & 10 \\
\hline \multicolumn{2}{|c|}{ Total weight } & \multicolumn{2}{|c|}{489.1} & 0.05 & \multicolumn{2}{|c|}{489.1} & 0.05 & 5 \\
\hline \multicolumn{2}{|c|}{$x$ c.g. location } & \multicolumn{2}{|c|}{165.3} & 0.04 & \multicolumn{2}{|c|}{165.3} & 0.04 & 5 \\
\hline \multicolumn{2}{|c|}{$y$ c.g. location } & \multicolumn{2}{|c|}{0.2} & -41.5 & 0.2 & & -41.5 & -- \\
\hline z c.g. lo & cation & 101. & & N/A & 101. & & N/A & -- \\
\hline
\end{tabular}

weight (1) for a total of 156 constraint functions. Again, the DOT algorithm was used for this optimization procedure. For both weight configurations, constraint value limits of $15 \%$ were used for off-diagonal terms $12-13,12-14,13-12$, and $14-12$ of the orthogonality matrices and off-diagonal terms 12-12 (mode interchange), 12-14, 13-13 (mode interchange), and 14-13 of the cross-orthogonality matrices. For all other off-diagonal terms, $10 \%$ limit values were used.

\begin{tabular}{|c|c|c|c|c|c|c|c|c|}
\hline \multicolumn{9}{|c|}{ EFEW configuration } \\
\hline & Mode shape & SW1B & AW1B & SW1T & AW1T & SW2B & AMLGL & SMLGL \\
\hline Mode shape & Mode number & $7^{*}$ & 8 & 9 & 11 & $\mathbf{1 3}$ & $\mathbf{1 2}$ & 14 \\
\hline SW1B & $7^{* *}$ & $\mathbf{0 . 9 9}$ & 0.00 & 0.00 & 0.00 & 0.08 & 0.00 & 0.07 \\
\hline AW1B & 8 & 0.00 & $\mathbf{1 . 0 0}$ & 0.00 & 0.00 & 0.00 & 0.10 & 0.00 \\
\hline SW1T & 9 & 0.01 & 0.00 & $\mathbf{0 . 9 2}$ & 0.00 & 0.00 & 0.00 & 0.01 \\
\hline AW1T & 11 & 0.00 & 0.01 & 0.00 & $\mathbf{0 . 9 8}$ & 0.00 & 0.29 & 0.00 \\
\hline SW2B & 12 & 0.09 & 0.00 & 0.07 & 0.01 & $\mathbf{0 . 9 5}$ & 0.03 & 0.89 \\
\hline AMLGL & 13 & 0.00 & 0.06 & 0.00 & 0.38 & 0.03 & $\mathbf{0 . 9 6}$ & 0.03 \\
\hline SMLGL & 14 & 0.05 & 0.00 & 0.06 & 0.00 & 0.88 & 0.00 & $\mathbf{0 . 9 7}$ \\
\hline \multicolumn{7}{|c|}{ FFFW configuration } & & \\
\hline Mode shape & Mode number & SW1B & AW1B & SW1T & AW1T & SW2B & AMLGL & SMLGL \\
\hline SW1B & $7 * *$ & 8 & 9 & 11 & $\mathbf{1 3}$ & $\mathbf{1 2}$ & 14 \\
\hline AW1B & 8 & 0.99 & 0.00 & 0.02 & 0.00 & 0.08 & 0.00 & 0.03 \\
\hline SW1T & 9 & 0.04 & 0.00 & $\mathbf{0 . 9 5}$ & 0.00 & 0.00 & 0.00 & 0.02 \\
\hline AW1T & 11 & 0.00 & 0.04 & 0.00 & $\mathbf{0 . 9 3}$ & 0.01 & 0.16 & 0.01 \\
\hline SW2B & 12 & 0.07 & 0.00 & 0.04 & 0.00 & $\mathbf{0 . 9 9}$ & 0.00 & 0.34 \\
\hline AMLGL & 13 & 0.00 & 0.04 & 0.00 & 0.31 & 0.01 & $\mathbf{0 . 9 7}$ & 0.01 \\
\hline SMLGL & 14 & 0.03 & 0.00 & 0.02 & 0.00 & 0.84 & 0.03 & $\mathbf{0 . 6 1}$ \\
\hline
\end{tabular}

*: Nastran mode number

**: GVT mode number 


\begin{tabular}{|c|c|c|c|c|c|c|c|c|}
\hline \multicolumn{9}{|c|}{ EFEW configuration } \\
\hline & Mode shape & SW1B & AW1B & SW1T & AW1T & SW2B & AMLGL & SMLGL \\
\hline Mode shape & Mode number & $7^{* *}$ & 8 & 9 & 11 & 12 & 13 & 14 \\
\hline SW1B & $7^{* *}$ & $\mathbf{1 . 0 0 0}$ & -0.022 & -0.054 & -0.011 & 0.030 & 0.025 & -0.038 \\
\hline AW1B & 8 & -0.022 & $\mathbf{1 . 0 0 0}$ & 0.013 & 0.000 & 0.007 & 0.034 & 0.002 \\
\hline SW1T & 9 & -0.054 & 0.013 & $\mathbf{1 . 0 0 0}$ & 0.004 & 0.036 & -0.009 & -0.025 \\
\hline AW1T & 11 & -0.011 & 0.000 & 0.004 & $\mathbf{1 . 0 0 0}$ & 0.020 & -0.082 & 0.002 \\
\hline SW2B & 12 & 0.030 & 0.007 & 0.036 & 0.020 & $\mathbf{1 . 0 0 0}$ & -0.150 & 0.107 \\
\hline AMLGL & 13 & 0.025 & 0.034 & -0.009 & -0.082 & -0.150 & $\mathbf{1 . 0 0 0}$ & 0.001 \\
\hline SMLGL & 14 & -0.038 & 0.002 & -0.025 & 0.002 & 0.107 & 0.001 & $\mathbf{1 . 0 0 0}$ \\
\hline \hline \multicolumn{7}{|c|}{ FFFW configuration } & & \\
\hline \\
\hline Mode shape & Mode number & $7 * *$ & 8 & 9 & 11 & 12 & 13 & 14 \\
\hline SW1B & $7 * *$ & $\mathbf{1 . 0 0 0}$ & 0.014 & -0.048 & 0.015 & 0.022 & 0.008 & -0.069 \\
\hline AW1B & 8 & 0.014 & $\mathbf{1 . 0 0 0}$ & -0.010 & 0.013 & -0.006 & -0.060 & -0.011 \\
\hline SW1T & 9 & -0.048 & -0.010 & $\mathbf{1 . 0 0 0}$ & -0.019 & 0.008 & -0.015 & -0.026 \\
\hline AW1T & 11 & 0.015 & 0.013 & -0.019 & $\mathbf{1 . 0 0 0}$ & -0.026 & 0.085 & 0.018 \\
\hline SW2B & 12 & 0.022 & -0.006 & 0.008 & -0.026 & $\mathbf{1 . 0 0 0}$ & -0.002 & 0.118 \\
\hline AMLGL & 13 & 0.008 & -0.060 & -0.015 & 0.085 & -0.002 & $\mathbf{1 . 0 0 0}$ & -0.076 \\
\hline SMLGL & 14 & -0.069 & -0.011 & -0.026 & 0.018 & 0.118 & -0.076 & $\mathbf{1 . 0 0 0}$ \\
\hline
\end{tabular}

**: GVT mode number

Fig. 26 Orthogonality matrix from previous history.

\begin{tabular}{|c|c|c|c|c|c|c|c|c|}
\hline \multicolumn{9}{|c|}{ EFEW configuration } \\
\hline & Mode shape & SW1B & AW1B & SW1T & AW1T & SW2B & AMLGL & SMLGL \\
\hline Mode shape & Mode number & $7^{*}$ & 8 & 9 & 11 & 13 & 12 & 14 \\
\hline SW1B & $7 * *$ & -1.007 & 0.015 & -0.036 & 0.005 & -0.061 & -0.013 & 0.047 \\
\hline AW1B & 8 & 0.007 & -1.017 & 0.004 & 0.002 & -0.003 & -0.038 & -0.005 \\
\hline SW1T & 9 & 0.028 & -0.008 & 1.043 & -0.015 & 0.069 & 0.009 & 0.077 \\
\hline AW1T & 11 & 0.000 & -0.005 & 0.008 & -1.032 & -0.006 & 0.030 & -0.003 \\
\hline SW2B & 12 & 0.002 & -0.008 & 0.119 & 0.001 & -0.927 & 0.238 & -0.139 \\
\hline AMLGL & 13 & -0.010 & -0.003 & 0.014 & 0.097 & -0.112 & -0.977 & -0.067 \\
\hline SMLGL & 14 & -0.012 & -0.004 & 0.072 & 0.012 & 0.000 & 0.078 & -0.894 \\
\hline \multicolumn{9}{|c|}{ FFFW configuration } \\
\hline 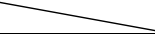 & Mode shape & SW1B & AW1B & SW1T & AW1T & SW2B & AMLGL & SMLGL \\
\hline Mode shape & Mode number & 7* & 8 & 9 & 11 & 13 & 12 & 14 \\
\hline SW1B & $7 * *$ & -1.084 & 0.008 & -0.038 & 0.011 & 0.040 & 0.008 & -0.065 \\
\hline AW1B & 8 & -0.004 & 1.107 & -0.008 & 0.019 & -0.007 & -0.072 & -0.012 \\
\hline SW1T & 9 & 0.026 & -0.005 & 1.151 & -0.013 & -0.103 & -0.008 & -0.026 \\
\hline AW1T & 11 & 0.018 & -0.004 & -0.035 & 1.077 & 0.016 & 0.006 & 0.007 \\
\hline SW2B & 12 & 0.011 & -0.005 & 0.106 & -0.001 & 1.054 & -0.097 & -0.162 \\
\hline AMLGL & 13 & 0.002 & -0.002 & 0.001 & 0.122 & 0.110 & 1.018 & 0.005 \\
\hline SMLGL & 14 & 0.008 & -0.012 & 0.042 & 0.022 & 0.286 & -0.138 & 0.884 \\
\hline
\end{tabular}

*: Nastran mode number

**: GVT mode number

Fig. 27 Cross-orthogonality matrix from previous history.

Table 6 also shows the frequency results for the third model-tuning procedure. The MAC matrix after the third model-tuning procedure is displayed in Fig. 28. MAC terms for mode number 14 improved for both weight configurations. Mass orthogonality matrix results are shown in Fig. 29. Small improvements were observed for the EFEW configuration with the off-diagonal term $12-13$ going from -0.150 to -0.142 and the off-diagonal term $12-14$ reducing from 0.107 to 0.091. Similarly, the off-diagonal term for the FFFW configuration went from 0.118 to 0.105 . Cross-orthogonality matrix results are shown in Fig. 30. Both objective function terms improved, with the EFEW mode interchanged off-diagonal term 12-12 improving the most from an initial value of 0.238 to 0.175 , whereas the FFFW mode interchanged off-diagonal term 14-13 going slightly bad from 0.286 to 0.288 .

\section{Fourth Model-Tuning Procedure}

Based on frequency results after the third model-tuning procedure in Table $\underline{6}$, the frequency result for mode number 28 of the EFEW configuration stood out due to the fact that frequency error was allowed to be at the maximum value of $20 \%$. For this model-tuning procedure, the goal was to improve this particular secondary mode frequency, and as a result, the frequency of the EFEW mode number 28 was selected as the objective function for this run. Similar to the last procedure, identical design variables were used with constraint functions consisting of all other cross-orthogonality matrix terms $(42+42)$, mass orthogonality matrix terms $(21+21)$, frequency terms $(14+12)$, total weight $(1+1), x$ c.g. locations $(1+1)$, and GVT sensor cable weight (1), for a total of 157 constraint functions. The DOT algorithm was used again for this optimization procedure.

Frequency results for the fourth model-tuning procedure are presented in Table $\underline{6}$. Notice that the frequency for the EFEW mode number 28, which was the objective function for this run, improved from $-20 \%$ from the previous procedure to $-16.7 \%$. MAC results for this procedure are shown in Fig. 31; a slight improvement was observed for the FFFW weight configuration, where mode number 14 went from 0.69 in the last procedure to 0.70 for this run. Figure $\underline{32}$ 
EFEW configuration

\begin{tabular}{|c|c|c|c|c|c|c|c|c|}
\hline \multicolumn{9}{|c|}{ EFEW configuration } \\
\hline & Mode shape & SW1B & AW1B & SW1T & AW1T & SW2B & AMLGL & SMLGL \\
\hline Mode shape & Mode number & $7^{*}$ & 8 & 9 & 11 & $\mathbf{1 3}$ & $\mathbf{1 2}$ & 14 \\
\hline SW1B & $7^{* *}$ & $\mathbf{0 . 9 9}$ & 0.00 & 0.00 & 0.00 & 0.08 & 0.00 & 0.07 \\
\hline AW1B & 8 & 0.00 & $\mathbf{1 . 0 0}$ & 0.00 & 0.01 & 0.00 & 0.09 & 0.00 \\
\hline SW1T & 9 & 0.01 & 0.00 & $\mathbf{0 . 9 3}$ & 0.00 & 0.00 & 0.00 & 0.00 \\
\hline AW1T & 11 & 0.00 & 0.01 & 0.00 & $\mathbf{0 . 9 9}$ & 0.01 & 0.31 & 0.01 \\
\hline SW2B & 12 & 0.09 & 0.00 & 0.06 & 0.01 & $\mathbf{0 . 9 7}$ & 0.01 & 0.89 \\
\hline AMLGL & 13 & 0.00 & 0.07 & 0.00 & 0.34 & 0.02 & $\mathbf{0 . 9 8}$ & 0.03 \\
\hline SMLGL & 14 & 0.05 & 0.00 & 0.05 & 0.00 & 0.89 & 0.00 & $\mathbf{0 . 9 8}$ \\
\hline \hline \multicolumn{7}{|c|}{ FFFW configuration } \\
\hline Mode shape & Mode number & $7^{*}$ & 8 & 9 & 11 & $\mathbf{1 3}$ & $\mathbf{1 2}$ & 14 \\
\hline SW1B & $7 * *$ & $\mathbf{0 . 9 9}$ & 0.00 & 0.03 & 0.00 & 0.08 & 0.01 & 0.03 \\
\hline AW1B & 8 & 0.00 & $\mathbf{1 . 0 0}$ & 0.00 & 0.03 & 0.00 & 0.06 & 0.00 \\
\hline SW1T & 9 & 0.04 & 0.00 & $\mathbf{0 . 9 6}$ & 0.00 & 0.00 & 0.00 & 0.02 \\
\hline AW1T & 11 & 0.00 & 0.03 & 0.00 & $\mathbf{0 . 9 5}$ & 0.03 & 0.15 & 0.02 \\
\hline SW2B & 12 & 0.07 & 0.00 & 0.03 & 0.00 & $\mathbf{0 . 9 8}$ & 0.05 & 0.43 \\
\hline AMLGL & 13 & 0.00 & 0.04 & 0.00 & 0.28 & 0.00 & $\mathbf{0 . 9 6}$ & 0.01 \\
\hline SMLGL & 14 & 0.03 & 0.00 & 0.02 & 0.00 & 0.86 & 0.01 & $\mathbf{0 . 6 9}$ \\
\hline
\end{tabular}

*: Nastran mode number

**: GVT mode number

Fig. 28 MAC matrix after the third model-tuning procedure (DOT-03).

\begin{tabular}{|c|c|c|c|c|c|c|c|c|}
\hline \multicolumn{9}{|c|}{ EFEW configuration } \\
\hline & Mode shape & SW1B & AW1B & SW1T & AW1T & SW2B & AMLGL & SMLGL \\
\hline Mode shape & Mode number & $7 * *$ & 8 & 9 & 11 & 12 & 13 & 14 \\
\hline SW1B & $7 * *$ & 1.000 & -0.016 & -0.053 & -0.006 & 0.035 & 0.022 & -0.042 \\
\hline AW1B & 8 & -0.016 & 1.000 & 0.005 & -0.002 & 0.003 & 0.032 & 0.003 \\
\hline SW1T & 9 & -0.053 & 0.005 & 1.000 & -0.004 & 0.033 & -0.001 & -0.026 \\
\hline AW1T & 11 & -0.006 & -0.002 & -0.004 & 1.000 & 0.007 & -0.075 & -0.002 \\
\hline SW2B & 12 & 0.035 & 0.003 & 0.033 & 0.007 & 1.000 & -0.142 & 0.091 \\
\hline AMLGL & 13 & 0.022 & 0.032 & -0.001 & -0.075 & -0.142 & 1.000 & 0.005 \\
\hline SMLGL & 14 & -0.042 & 0.003 & -0.026 & -0.002 & 0.091 & 0.005 & 1.000 \\
\hline \multicolumn{9}{|c|}{ FFFW configuration } \\
\hline & Mode shape & SW1B & AW1B & SW1T & AW1T & SW2B & AMLGL & SMLGL \\
\hline Mode shape & Mode number & $7 * *$ & 8 & 9 & 11 & 12 & 13 & 14 \\
\hline SW1B & $7 * *$ & 1.000 & 0.008 & -0.046 & 0.009 & 0.025 & 0.006 & -0.071 \\
\hline AW1B & 8 & 0.008 & 1.000 & -0.003 & 0.012 & -0.001 & -0.060 & -0.011 \\
\hline SW1T & 9 & -0.046 & -0.003 & 1.000 & -0.012 & 0.005 & -0.008 & -0.026 \\
\hline AW1T & 11 & 0.009 & 0.012 & -0.012 & 1.000 & -0.016 & 0.078 & 0.019 \\
\hline SW2B & 12 & 0.025 & -0.001 & 0.005 & -0.016 & 1.000 & 0.009 & 0.105 \\
\hline AMLGL & 13 & 0.006 & -0.060 & -0.008 & 0.078 & 0.009 & 1.000 & -0.072 \\
\hline SMLGL & 14 & -0.071 & -0.011 & -0.026 & 0.019 & 0.105 & -0.072 & 1.000 \\
\hline
\end{tabular}

**: GVT mode number

Fig. 29 Orthogonality matrix after the third model-tuning procedure (DOT-03).

\begin{tabular}{|c|c|c|c|c|c|c|c|c|}
\hline \multicolumn{9}{|c|}{ EFEW configuration } \\
\hline & Mode shape & SW1B & AW1B & SW1T & AW1T & SW2B & AMLGL & SMLGL \\
\hline Mode shape & Mode number & $7^{*}$ & 8 & 9 & 11 & $\mathbf{1 3}$ & $\mathbf{1 2}$ & 14 \\
\hline SW1B & $7^{* *}$ & $\mathbf{1 . 0 0 1}$ & -0.016 & -0.037 & 0.010 & -0.064 & -0.014 & 0.051 \\
\hline AW1B & 8 & 0.001 & $\mathbf{1 . 0 1 7}$ & 0.005 & -0.005 & 0.001 & -0.041 & -0.003 \\
\hline SW1T & 9 & -0.026 & -0.002 & $\mathbf{1 . 0 3 6}$ & -0.047 & 0.048 & 0.013 & 0.067 \\
\hline AW1T & 11 & 0.006 & -0.001 & -0.037 & $\mathbf{- 1 . 0 3 4}$ & -0.023 & 0.065 & -0.016 \\
\hline SW2B & 12 & -0.004 & 0.007 & 0.099 & 0.023 & $\mathbf{- 0 . 9 5 5}$ & 0.175 & -0.140 \\
\hline AMLGL & 13 & 0.010 & -0.002 & 0.024 & 0.052 & -0.057 & $\mathbf{- 1 . 0 0 4}$ & -0.034 \\
\hline SMLGL & 14 & 0.015 & 0.003 & 0.064 & 0.023 & 0.008 & 0.037 & $\mathbf{- 0 . 9 4 3}$ \\
\hline \hline \multicolumn{7}{|c|}{ FFFW configuration } \\
\hline Mode shape & Mode number & $7^{*}$ & 8 & 9 & 11 & $\mathbf{1 3}$ & $\mathbf{1 2}$ & 14 \\
\hline SW1B & $7 * *$ & $\mathbf{- 1 . 0 8 1}$ & 0.009 & 0.039 & 0.019 & 0.039 & -0.017 & -0.070 \\
\hline AW1B & 8 & 0.004 & $\mathbf{1 . 1 0 6}$ & 0.009 & 0.027 & 0.012 & 0.074 & -0.011 \\
\hline SW1T & 9 & 0.024 & 0.005 & $\mathbf{- 1 . 1 4 6}$ & -0.036 & -0.077 & 0.026 & -0.029 \\
\hline AW1T & 11 & 0.025 & -0.010 & 0.001 & $\mathbf{1 . 0 9 3}$ & -0.012 & -0.041 & 0.004 \\
\hline SW2B & 12 & 0.011 & -0.001 & -0.084 & 0.014 & $\mathbf{1 . 0 5 0}$ & -0.152 & -0.151 \\
\hline AMLGL & 13 & 0.003 & 0.001 & -0.009 & 0.080 & -0.121 & $\mathbf{- 1 . 0 3 3}$ & 0.000 \\
\hline SMLGL & 14 & 0.005 & -0.012 & -0.037 & 0.032 & 0.288 & 0.062 & $\mathbf{0 . 9 2 5}$ \\
\hline
\end{tabular}

*: Nastran mode number

**: GVT mode number

Fig. 30 Cross-orthogonality matrix after the third model-tuning procedure (DOT-03). 


\begin{tabular}{|c|c|c|c|c|c|c|c|c|}
\hline \multicolumn{9}{|c|}{ EFEW configuration } \\
\hline & Mode shape & SW1B & AW1B & SW1T & AW1T & SW2B & AMLGL & SMLGL \\
\hline Mode shape & Mode number & $7 *$ & 8 & 9 & 11 & $\mathbf{1 3}$ & $\mathbf{1 2}$ & 14 \\
\hline SW1B & $7^{* *}$ & $\mathbf{0 . 9 9}$ & 0.00 & 0.00 & 0.00 & 0.08 & 0.00 & 0.06 \\
\hline AW1B & 8 & 0.00 & $\mathbf{1 . 0 0}$ & 0.00 & 0.01 & 0.00 & 0.09 & 0.00 \\
\hline SW1T & 9 & 0.01 & 0.00 & $\mathbf{0 . 9 3}$ & 0.00 & 0.00 & 0.00 & 0.00 \\
\hline AW1T & 11 & 0.00 & 0.01 & 0.00 & $\mathbf{0 . 9 9}$ & 0.01 & 0.30 & 0.01 \\
\hline SW2B & 12 & 0.09 & 0.00 & 0.05 & 0.01 & $\mathbf{0 . 9 7}$ & 0.01 & 0.89 \\
\hline AMLGL & 13 & 0.00 & 0.07 & 0.00 & 0.35 & 0.02 & $\mathbf{0 . 9 8}$ & 0.03 \\
\hline SMLGL & 14 & 0.05 & 0.00 & 0.04 & 0.00 & 0.89 & 0.00 & $\mathbf{0 . 9 8}$ \\
\hline \hline \multicolumn{7}{|c|}{ FFFW configuration } \\
\hline Mode shape & Mode number & $7 *$ & 8 & 9 & 11 & $\mathbf{1 3}$ & $\mathbf{1 2}$ & 14 \\
\hline SW1B & $7 * *$ & $\mathbf{0 . 9 9}$ & 0.00 & 0.03 & 0.00 & 0.08 & 0.01 & 0.03 \\
\hline AW1B & 8 & 0.00 & $\mathbf{1 . 0 0}$ & 0.00 & 0.03 & 0.00 & 0.06 & 0.00 \\
\hline SW1T & 9 & 0.04 & 0.00 & $\mathbf{0 . 9 6}$ & 0.00 & 0.00 & 0.00 & 0.02 \\
\hline AW1T & 11 & 0.00 & 0.03 & 0.00 & $\mathbf{0 . 9 5}$ & 0.03 & 0.14 & 0.02 \\
\hline SW2B & 12 & 0.07 & 0.00 & 0.02 & 0.00 & $\mathbf{0 . 9 8}$ & 0.05 & 0.44 \\
\hline AMLGL & 13 & 0.00 & 0.04 & 0.00 & 0.27 & 0.00 & $\mathbf{0 . 9 6}$ & 0.01 \\
\hline SMLGL & 14 & 0.03 & 0.00 & 0.01 & 0.00 & 0.86 & 0.01 & $\mathbf{0 . 7 0}$ \\
\hline
\end{tabular}

*: Nastran mode number

**: GVT mode number

Fig. 31 MAC matrix after the fourth model-tuning procedure (DOT-04).

\begin{tabular}{|c|c|c|c|c|c|c|c|c|}
\hline \multicolumn{9}{|c|}{ EFEW configuration } \\
\hline & Mode shape & SW1B & AW1B & SW1T & AW1T & SW2B & AMLGL & SMLGL \\
\hline Mode shape & Mode number & $7 * *$ & 8 & 9 & 11 & 12 & 13 & 14 \\
\hline SW1B & $7 * *$ & 1.000 & -0.016 & -0.052 & -0.006 & 0.035 & 0.022 & -0.040 \\
\hline AW1B & 8 & -0.016 & 1.000 & 0.005 & -0.001 & 0.002 & 0.035 & 0.002 \\
\hline SW1T & 9 & -0.052 & 0.005 & 1.000 & -0.003 & 0.031 & -0.001 & -0.026 \\
\hline AW1T & 11 & -0.006 & -0.001 & -0.003 & 1.000 & 0.009 & -0.077 & -0.001 \\
\hline SW2B & 12 & 0.035 & 0.002 & 0.031 & 0.009 & 1.000 & -0.141 & 0.110 \\
\hline AMLGL & 13 & 0.022 & 0.035 & -0.001 & -0.077 & -0.141 & 1.000 & 0.007 \\
\hline SMLGL & 14 & -0.040 & 0.002 & -0.026 & -0.001 & 0.110 & 0.007 & 1.000 \\
\hline \multicolumn{9}{|c|}{ FFFW configuration } \\
\hline ( & Mode shape & SW1B & AW1B & SW1T & AW1T & SW2B & AMLGL & SMLGL \\
\hline Mode shape & Mode number & $7 * *$ & 8 & 9 & 11 & 12 & 13 & 14 \\
\hline SW1B & $7 * *$ & 1.000 & 0.009 & -0.046 & 0.010 & 0.026 & 0.006 & -0.070 \\
\hline AW1B & 8 & 0.009 & 1.000 & -0.003 & 0.012 & -0.001 & -0.061 & -0.011 \\
\hline SW1T & 9 & -0.046 & -0.003 & 1.000 & -0.012 & 0.005 & -0.009 & -0.026 \\
\hline AW1T & 11 & 0.010 & 0.012 & -0.012 & 1.000 & -0.017 & 0.080 & 0.019 \\
\hline SW2B & 12 & 0.026 & -0.001 & 0.005 & -0.017 & 1.000 & 0.010 & 0.120 \\
\hline AMLGL & 13 & 0.006 & -0.061 & -0.009 & 0.080 & 0.010 & 1.000 & -0.073 \\
\hline SMLGL & 14 & -0.070 & -0.011 & -0.026 & 0.019 & 0.120 & -0.073 & 1.000 \\
\hline
\end{tabular}

**: GVT mode number

Fig. 32 Orthogonality matrix after the fourth model-tuning procedure (DOT-04).

\begin{tabular}{|c|c|c|c|c|c|c|c|c|}
\hline \multicolumn{9}{|c|}{ EFEW configuration } \\
\hline & Mode shape & SW1B & AW1B & SW1T & AW1T & SW2B & AMLGL & SMLGL \\
\hline Mode shape & Mode number & $7^{*}$ & 8 & 9 & 11 & $\mathbf{1 3}$ & $\mathbf{1 2}$ & 14 \\
\hline SW1B & $7^{* *}$ & $\mathbf{- 1 . 0 0 0}$ & -0.016 & 0.038 & 0.009 & -0.062 & -0.014 & 0.049 \\
\hline AW1B & 8 & 0.000 & $\mathbf{1 . 0 1 7}$ & -0.005 & -0.005 & 0.000 & -0.038 & -0.003 \\
\hline SW1T & 9 & 0.024 & -0.002 & $\mathbf{- 1 . 0 3 5}$ & -0.044 & 0.041 & 0.012 & 0.062 \\
\hline AW1T & 11 & -0.006 & -0.002 & 0.032 & $\mathbf{- 1 . 0 3 3}$ & -0.022 & 0.060 & -0.015 \\
\hline SW2B & 12 & 0.002 & 0.006 & -0.093 & 0.023 & $\mathbf{- 0 . 9 4 9}$ & 0.175 & -0.141 \\
\hline AMLGL & 13 & -0.010 & 0.004 & -0.022 & 0.056 & -0.057 & $\mathbf{- 0 . 9 9 9}$ & -0.034 \\
\hline SMLGL & 14 & -0.014 & 0.002 & -0.059 & 0.023 & -0.004 & 0.037 & $\mathbf{- 0 . 9 3 2}$ \\
\hline \hline \multicolumn{7}{|c|}{ FFFW configuration } & & \\
\hline Mode shape & Mode number & SW1B & AW1B & SW1T & AW1T & SW2B & AMLGL & SMLGL \\
\hline SW1B & $7 * *$ & 8 & 9 & 11 & $\mathbf{1 3}$ & $\mathbf{1 2}$ & 14 \\
\hline AW1B & 8 & $-\mathbf{1 . 0 7 9}$ & 0.009 & 0.040 & 0.017 & -0.039 & -0.017 & 0.067 \\
\hline SW1T & 9 & 0.003 & $\mathbf{1 . 1 0 6}$ & 0.009 & 0.026 & -0.012 & 0.070 & 0.011 \\
\hline AW1T & 11 & 0.025 & -0.004 & $\mathbf{- 1 . 1 4 6}$ & -0.034 & 0.068 & 0.024 & 0.029 \\
\hline SW2B & 12 & 0.010 & -0.001 & -0.077 & 0.015 & $\mathbf{- 1 . 0 4 9}$ & -0.153 & 0.140 \\
\hline AMLGL & 13 & 0.003 & -0.004 & -0.008 & 0.080 & 0.122 & $\mathbf{- 1 . 0 2 9}$ & 0.000 \\
\hline SMLGL & 14 & 0.006 & -0.011 & -0.033 & 0.031 & -0.286 & 0.059 & $\mathbf{- 0 . 9 1 1}$ \\
\hline
\end{tabular}

*: Nastran mode number

**: GVT mode number

Fig. 33 Cross-orthogonality matrix after the fourth model-tuning procedure (DOT-04). 


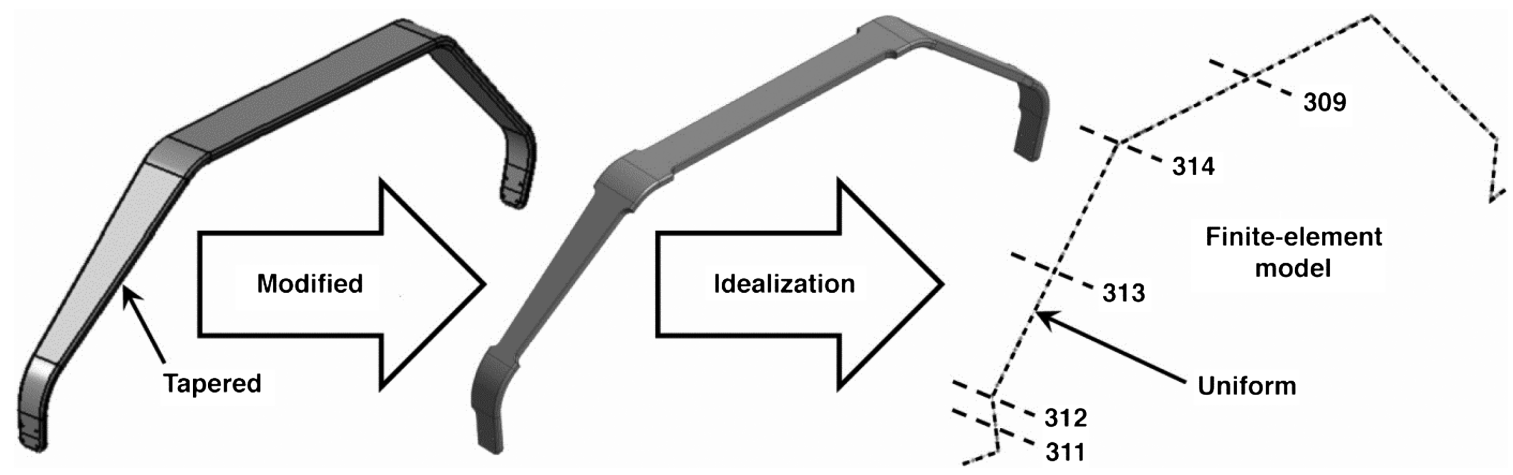

Fig. 34 Main landing gear model.

shows the results of the mass orthogonality matrix; only a slight improvement was seen for the off-diagonal term 12-13 for the EFEW configuration. The off-diagonal term 12-14 for the EFEW and FFFW increased from 0.091 to 0.110 and from 0.105 to 0.120 , respectively. Cross-orthogonality matrix results are displayed in Fig. 33. Not much change was observed between the last run and this run, with the exception of the off-diagonal term 12-14 for the FFFW weight configuration, which went from a magnitude of 0.151 to 0.140 .

The design variable changes of the model-tuning procedures are summarized in Table 7, which actually displays the design variable percentage changes between each model-tuning procedure. Notice that the percentage change for many of the main landing gear design variables after the fourth model-tuning procedure (DOT-04) approached the $\pm 20 \%$ side constraint limit. In addition, the smallest MAC value, largest frequency error, largest off-diagonal terms of orthogonality, and cross-orthogonality matrices were all related to the main landing gear. Therefore, it can be concluded that the FE model pertaining to the main landing gear was not accurate enough to allow significant change or improvement. It was later found that there was an idealization error with the main landing gear. This reasoning for this idealization error is presented in Fig. 34. In Fig. 34, the main landing-gear was originally designed as tapered and curved beams. When creating the FE model, the tapered and curved regions of the main landing gear were modeled as uniform and straight beams, as

Table 6 Flexible modes from previous history, and after third and fourth model-tuning procedures

\begin{tabular}{|c|c|c|c|c|c|c|c|c|c|c|c|}
\hline & & \multicolumn{9}{|c|}{ Nastran results } & \multirow[b]{3}{*}{ Target errol } \\
\hline \multicolumn{2}{|c|}{ GVT data } & \multicolumn{3}{|c|}{$\mathrm{POH}$} & \multicolumn{3}{|c|}{ DOT-03 } & \multicolumn{3}{|c|}{ DOT-04 } & \\
\hline Mode & Mode shape & Mode & Frequency & Error, $\%$ & Mode & Frequency & Error & Mode & Frequency & Error & \\
\hline \multicolumn{12}{|c|}{ EFEW configuration } \\
\hline 7 & SW1B & 7 & 1.086 & 1.8 & 7 & 1.090 & 2.2 & 7 & 1.101 & 3.1 & $5(3)$ \\
\hline 8 & AW1B & 8 & 1.543 & 0.0 & 8 & 1.549 & 0.4 & 8 & 1.565 & 1.5 & $5(3)$ \\
\hline 9 & SW1T & 9 & 3.276 & 1.6 & 9 & 3.256 & 1.0 & 9 & 3.294 & 2.2 & $5(3)$ \\
\hline 11 & AW1T & 11 & 3.823 & -0.4 & 11 & 3.778 & -1.6 & 11 & 3.834 & -0.1 & $5(3)$ \\
\hline 12 & SW2B & 13 & 4.642 & 4.6 & 13 & 4.611 & 3.9 & 13 & 4.662 & 5.0 & 5 \\
\hline 13 & AMLGL & 12 & 4.415 & -1.2 & 12 & 4.401 & -1.5 & 12 & 4.460 & -0.1 & $5(3)$ \\
\hline 14 & SMLGL & 14 & 4.715 & 1.1 & 14 & 4.683 & 0.4 & 14 & 4.738 & 1.5 & $5(3)$ \\
\hline 15 & BoomH & 15 & 5.217 & -1.1 & 15 & 5.219 & -1.0 & 15 & 5.222 & -1.0 & $10(3)$ \\
\hline 18 & AW2B & 18 & 6.106 & 1.3 & 18 & 6.105 & 1.3 & 18 & 6.149 & 2.0 & 10(3) \\
\hline 19 & SWL & 19 & 6.242 & -0.4 & 19 & 6.246 & -0.3 & 19 & 6.270 & 0.1 & $10(3)$ \\
\hline 25 & SW3B & 25 & 9.473 & 1.4 & 25 & 9.479 & 1.4 & 25 & 9.539 & 2.1 & 10(3) \\
\hline 26 & AW3B & 27 & 11.01 & 3.9 & 27 & 11.22 & -1.4 & 27 & 11.59 & 2.0 & $10(3)$ \\
\hline 28 & AMLGFA & 26 & 9.544 & -20.0 & 26 & 9.544 & -20.0 & 26 & 9.938 & -16.7 & 20 \\
\hline 30 & AW2T & 30 & 13.09 & 5.5 & 30 & 13.04 & 5.1 & 30 & 13.14 & 6.0 & 10 \\
\hline \multicolumn{2}{|c|}{ Total weight } & \multicolumn{2}{|r|}{368.1} & 0.37 & \multicolumn{2}{|r|}{367.7} & 0.28 & \multicolumn{2}{|r|}{367.4} & 0.20 & 5 \\
\hline \multicolumn{2}{|c|}{$x$ c.g. location } & \multicolumn{2}{|r|}{164.8} & -0.14 & \multicolumn{2}{|r|}{164.8} & -0.14 & \multicolumn{2}{|r|}{164.8} & -0.15 & 5 \\
\hline & & & 0.4 & -481 & \multicolumn{2}{|r|}{0.4} & -466 & \multicolumn{2}{|r|}{0.4} & -462 & -- \\
\hline \multicolumn{2}{|c|}{$\begin{array}{l}y \text { c.g. location } \\
z \text { c.g. location }\end{array}$} & \multicolumn{2}{|r|}{$\begin{array}{l}0.4 \\
101.7\end{array}$} & N/A & & 101.8 & N/A & \multirow{2}{*}{\multicolumn{2}{|c|}{101.8}} & N/A & -- \\
\hline & & & & & \multicolumn{3}{|c|}{ FFFW configuration } & & & & \\
\hline 7 & SW1B & 7 & 0.999 & -0.1 & 7 & 1.003 & 0.3 & 7 & 1.011 & 1.1 & $5(3)$ \\
\hline 8 & AW1B & 8 & 1.402 & -0.6 & 8 & 1.407 & -0.2 & 8 & 1.421 & 0.8 & $5(3)$ \\
\hline 9 & SW1T & 9 & 3.000 & 2.1 & 9 & 2.988 & 1.7 & 9 & 3.021 & 2.8 & $5(3)$ \\
\hline 11 & AW1T & 11 & 3.615 & -1.0 & 11 & 3.579 & -2.0 & 11 & 3.630 & -0.6 & $5(3)$ \\
\hline 12 & SW2B & 13 & 4.469 & 2.8 & 13 & 4.427 & 1.9 & 13 & 4.481 & 3.1 & $5(3)$ \\
\hline 13 & AMLGL & 12 & 4.357 & -1.1 & 12 & 4.343 & -1.5 & 12 & 4.401 & -0.1 & $5(3)$ \\
\hline 14 & SMLGL & 14 & 4.672 & 1.5 & 14 & 4.641 & 0.9 & 14 & 4.695 & 2.0 & $5(3)$ \\
\hline 16 & BoomH & 16 & 5.219 & -1.1 & 16 & 5.219 & -1.1 & 16 & 5.220 & -1.1 & $10(3)$ \\
\hline 19 & SWL & 19 & 6.060 & -1.4 & 19 & 6.068 & -1.2 & 19 & 6.090 & -0.9 & $10(3)$ \\
\hline 24 & SW3B & 24 & 8.745 & 1.0 & 24 & 8.748 & 1.1 & 24 & 8.808 & 1.8 & 10(3) \\
\hline 25 & NLGFA & 25 & 9.172 & 0.5 & 25 & 9.174 & 0.5 & 25 & 9.183 & 0.6 & 10(3) \\
\hline 28 & $\mathrm{AW} 2 \mathrm{~T}$ & 30 & 11.93 & 3.4 & 30 & 11.88 & 3.0 & 30 & 11.96 & 3.6 & $10(5)$ \\
\hline \multicolumn{2}{|c|}{ Total weight } & \multicolumn{2}{|c|}{491.1} & 0.46 & \multicolumn{2}{|r|}{490.8} & 0.39 & & 490.5 & 0.33 & 5 \\
\hline$x \mathrm{c}$ & location & & 165.3 & 0.06 & & 165.3 & 0.05 & & 165.3 & 0.05 & 5 \\
\hline$y \mathrm{c}$ & location & & 0.3 & -28.7 & & 0.3 & -31.5 & & 0.3 & -32.19 & -- \\
\hline$z \mathrm{c}$ & Location & & 101.3 & N/A & & 101.3 & N/A & & 101.4 & N/A & -- \\
\hline
\end{tabular}


Table 7 Design variable changes (percent) during model-tuning procedures

\begin{tabular}{|c|c|c|c|c|c|c|c|c|}
\hline & Design variable & Property & Base $^{\mathrm{a}}$ & DOT1 & DOT2 & $\mathrm{POH}^{\mathrm{b}}$ & DOT3 & DOT4 \\
\hline 1 & CONM2‘ 100202 and 100204 & Mass & 0.0 & 0.8 & 0.8 & 13.4 & 17.6 & 7.9 \\
\hline 2 & PBAR 310 & $I_{1}$ & 0.0 & 0.0 & 0.0 & 0.0 & 0.2 & 1.1 \\
\hline 3 & PBAR 313 & $I_{1}$ & 0.0 & -1.4 & -1.4 & -8.5 & 8.9 & 11.7 \\
\hline 4 & PBAR 314 & $I_{1}$ & 0.0 & -0.6 & -0.6 & -3.3 & -10.4 & -20.0 \\
\hline 5 & PBAR 308 & $I_{1}$ & 0.0 & -0.5 & -0.5 & -2.0 & -4.7 & -12.9 \\
\hline 6 & PBAR 310 & $I_{2}$ & 0.0 & 0.3 & 0.3 & -0.7 & -4.5 & -4.6 \\
\hline 7 & PBAR 313 & $I_{2}$ & 0.0 & 0.4 & 0.4 & 0.3 & 1.9 & 18.0 \\
\hline 8 & PBAR 310 & $\vec{J}$ & 0.0 & 0.0 & 0.0 & 0.0 & -0.1 & -0.1 \\
\hline 9 & PBAR 313 & $J$ & 0.0 & 0.1 & 0.1 & 0.1 & 2.6 & 9.2 \\
\hline 10 & PBAR 314 & $J$ & 0.0 & 0.4 & 0.4 & 0.4 & 6.4 & 20.0 \\
\hline 11 & PBUSH $^{\text {e }} 315$ and 316 & $K_{2}$ & 0.0 & 0.0 & 0.0 & -0.5 & -0.1 & -1.6 \\
\hline 12 & PBUSH 315 and 316 & $K_{3}$ & 0.0 & 0.0 & 0.0 & -0.7 & -6.7 & -4.2 \\
\hline 13 & PBUSH 315 and 316 & $K_{4}$ & 0.0 & 0.0 & 0.0 & -0.5 & -2.3 & -4.9 \\
\hline 14 & MAT8 100 & $E$ & 0.0 & -2.0 & -2.0 & 0.7 & 1.5 & 4.8 \\
\hline 15 & MAT8 100 & $G$ & 0.0 & 6.4 & 6.4 & 9.0 & 13.9 & 17.9 \\
\hline 16 & PBAR 311 & $A$ & 0.0 & 0.0 & 0.0 & 0.4 & 1.0 & 1.1 \\
\hline 17 & PBAR 312 & $A$ & 0.0 & 0.0 & 0.0 & 2.3 & 9.0 & 15.3 \\
\hline 18 & PBAR 313 & $A$ & 0.0 & 0.0 & 0.0 & 1.3 & 9.9 & 17.1 \\
\hline 19 & PBAR 312 & $I_{1}$ & 0.0 & 0.0 & 0.0 & -0.1 & 0.5 & 1.0 \\
\hline 20 & PBAR 309 & $I_{1}$ & 0.0 & 0.0 & 0.0 & -1.6 & -6.3 & -17.2 \\
\hline 21 & CONM2 930618 & Mass & 0.0 & 0.0 & 0.0 & 0.0 & -1.9 & -2.2 \\
\hline 22 & CONM2 9120105 & Mass & 0.0 & 0.0 & 0.0 & 1.2 & -20.0 & -20.0 \\
\hline 23 & CONM2 930668 & Mass & 0.0 & 0.0 & 0.0 & -0.1 & 2.0 & 2.5 \\
\hline 24 & CONM2 9220105 & Mass & 0.0 & 0.0 & 0.0 & -2.2 & 10.9 & 8.1 \\
\hline
\end{tabular}

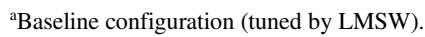

${ }^{\mathrm{b}}$ Obtained from previous optimization history.

${ }^{\mathrm{c} C}$ Concentrated mass element (MSC Nastran terminology).

${ }^{\mathrm{d}}$ Simple beam property (MSC Nastran terminology).

${ }^{\mathrm{e}}$ Generalized spring and damper property (MSC Nastran terminology).

${ }^{\mathrm{f}} \mathrm{S} h e l l$ element orthotropic material property (MSC Nastran terminology).

opposed to tapered and curved beams, respectively. Improving properties related to the main landing gear was not an easy task because only a single uniform sectional property was used for the whole tapered region. This was the main reason why some design variables in Table 7 stood out due to their large changes, but because a $\pm 20 \%$ side constraint was imposed and none of the design variables exceeded this side constraint margin, the resulting changes for the physical properties were deemed acceptable.

Nondimensional flutter speeds and frequencies after the fourth model tuning are summarized in Table 3 . Corresponding speed versus damping $V-g$ and speed versus frequency $V-f$ curves of this model with the EFEW configuration at a Mach number of 0.160 from the matched flutter analyses are given in Fig. 35. Improved frequencies and mode shapes in this study increased average flutter speeds by $7.6 \%$ compared to the baseline model. In Fig. 16, notice that LMSW's model tuning resulted in large increases in flutter speed for the baseline model when compared to their nonvalidated final design model. From this model-tuning procedure done by NASA, further inroads were made by increasing the flutter speed for the symmetric body freedom flutter and symmetric wing bending torsion flutter, as shown in Fig. 16.

\section{Conclusions}

For the X-56A flight-test support, NASA AFRC has undertaken the task of improving the GVT test-validated structural dynamic finiteelement model. Using an in-house $\mathrm{O}^{3}$ tool developed by NASA AFRC's structural dynamics group, along with the Nastran program, structural model tuning was conducted so as to improve the correlation between numerical and experimental modal data to reduce model uncertainties by meeting specified NASA and military standards.

Structural dynamic model tuning of the aircraft was performed in stages, starting with tuning of the frequency, followed by the crossorthogonality (mode shape) matrix. A total of four structural dynamic model tuning runs were conducted focusing on the improvement in frequency errors and the off-diagonal terms of the cross-orthogonality matrices for both the EFEW and the FFFW configurations of the X-56A aircraft in a single optimization run. Other properties such as weight of GVT sensor cables, total weight, c.g. location, and off-diagonal terms of the orthogonality matrix were primarily used as constraints because the current configurations met the majority of the defined criteria. MAC constraints were not used as objective or constraint functions. However, the MAC values were used in a mode-tracking program to overcome mode interchange problems during model-tuning procedure.

The first and second model-tuning procedures focused on the improvement of frequencies and off-diagonal terms of the crossorthogonality matrices to meet the NASA standard by using main landing gear, Young's modulus, and Shear modulus design variables. All of the off-diagonal terms violate military and NASA standards, which were subsequently improved after the first two model-tuning procedures. An average of $3.8 \%$ of improvement was observed in the cross-orthogonality matrices. Slight violation of target frequency values was also observed after the first two model-tuning procedures.

Before the third and fourth model-tuning procedures, previous optimization histories were reviewed, and a much better design configuration was found by change. By selecting an improved optimization configuration based on $\mathrm{POH}$ as the new starting point, the optimization results for the third and fourth model-tuning procedures were improved significantly. It should be noted that the largest correlation errors were mostly associated with the main landing gear due to the existence of the idealization error related to the main landing gear.

Finally, it should be emphasized that the performance indices based on each individual element of frequency errors and the offdiagonal terms of the orthogonality and cross-orthogonality matrices introduced in this study were easier to use than the norm averagebased model-tuning method $[2,17]$. In the case of the norm-based approach, the total number of performance indices was a lot smaller than the current method; however, it was not easy to control each individual off-diagonal term when compared to the current approach.

\section{Acknowledgments}

The work presented in this paper was funded by the Aero Science Project under the ARMD Fundamental Aeronautics Program. The 

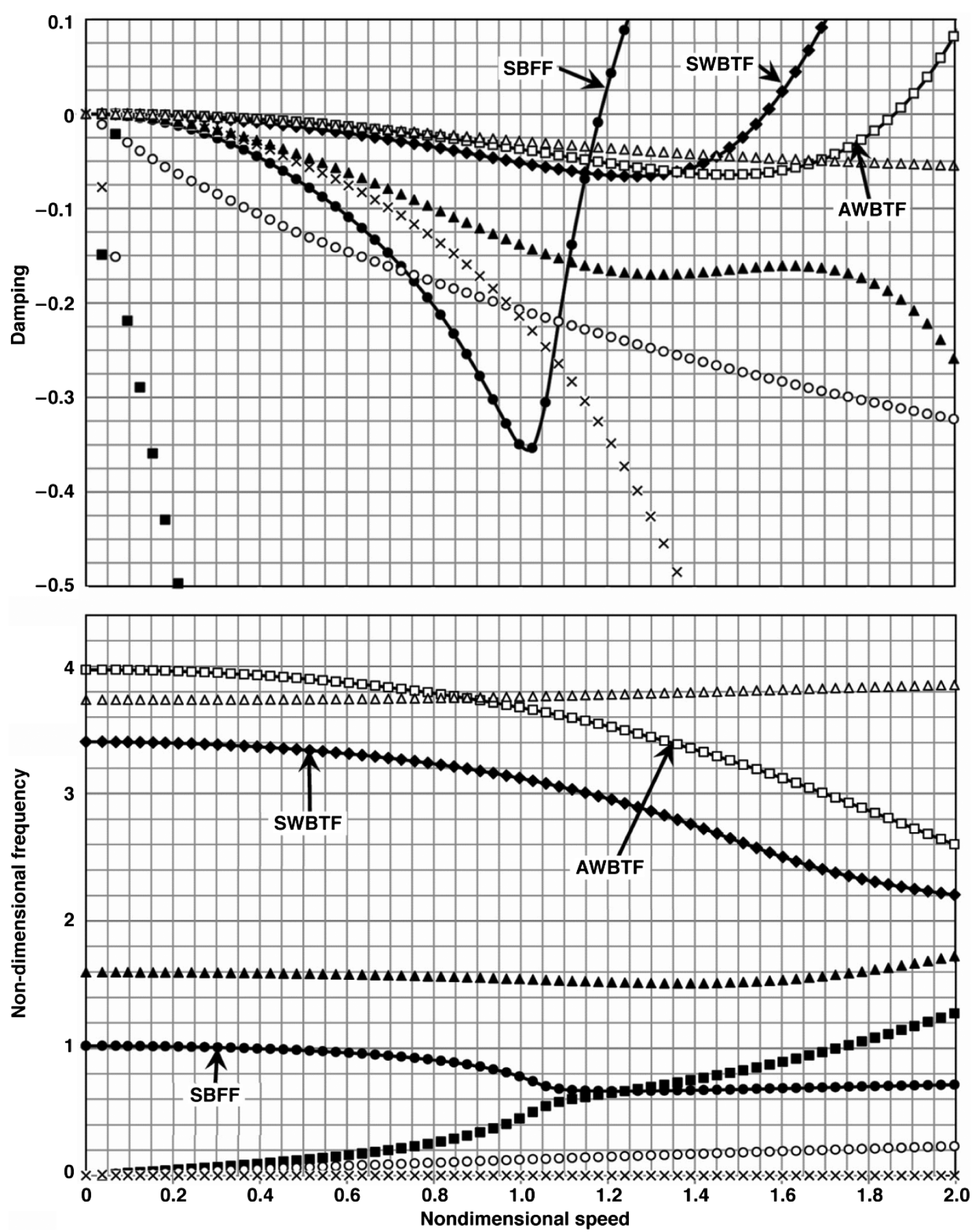

Fig. $35 V-g$ and $V-f$ curves after DOT-04 with the EFEW at Mach 0.16.

project managers at NASA AFRC were Mark Davis and Robert Navarro. The authors also thank Jeff Beranek at Lockheed Martin Skunk Works for his support in providing the finite-element models and the ground-vibration test data during this research. Finally, thanks to Pete Flick at the U.S. Air Force Research Laboratory for providing the flight hardware and test data to NASA.

\section{References}

[1] Beranek, J., Nicolai, L., Buonanno, M., Burnett, E., Atkinson, C., HolmHansen, B., and Flick, P., "Conceptual Design of a Multi-Utility Aeroelastic Demonstrator," 13th AIAA/ISSMO Multidisciplinary Analysis Optimization Conference, AIAA Paper 2010-9350, Sept. 2010.

[2] Pak, C.-G., and Lung, S.-F., "Flutter Analysis of the Aerostructures Test Wing with Test Validated Structural Dynamic Model," Journal of Aircraft, Vol. 48, No. 4, 2011, pp. 1263-1272. doi: $10.2514 / 1 . C 031257$
[3] Pak, C.-G., "Unsteady Aerodynamic Model Tuning for Precise Flutter Prediction," Journal of Aircraft, Vol. 48, No. 6, 2011, pp. 2178-2184. doi:10.2514/1.C031495

[4] Baruch, M., and Bar-Itzhack, I. Y., "Optimal Weighted Orthogonalization of Measured Modes," AIAA Journal, Vol. 16, No. 4, 1978, pp. 346351. doi: $10.2514 / 3.60896$

[5] Minas, C., and Inman, D. J., "Matching Finite Element Models to Modal Data," Journal of Vibration and Acoustics, Vol. 112, No. 1, 1990, pp. 84-92. doi:10.1115/1.2930103

[6] Berman, A., and Nagy, E. J., "Improvement of a Large Analytical Model Using Test Data," AIAA Journal, Vol. 21, No. 8, 1983, pp. 1168-1173. doi:10.2514/3.60140

[7] Schwarz, B., Richardson, M., and Formenti, D. L., "FEA Model Updating Using SDM," Sound and Vibration, Vol. 41, No. 10, 2007, pp. $18-23$.

[8] Heylen, W., and Vanhonacker, P., "An Automated Technique for Improving Model Matrices by Means of Experimentally Obtained 
Dynamic Data," 24th Structures, Structural Dynamics and Materials Conference, AIAA Paper 1983-0881, 1983.

[9] Juneja, V., Haftka, R. T., and Cudney, H. H., "Location of Damage in a Space Structure by Contrast Maximization," Dynamics Specialists Conference, AIAA Paper 1994-1711, 1994.

[10] Kim, H. M., and Bartkowicz, T. J., "Damage Detection and Health Monitoring of Large Space Structures," Sound and Vibration, Vol. 27, No. 6, 1993, pp. 12-17.

[11] Zimmerman, D. C., and Simmermacher, T, "Model Refinement and System Health Monitoring Using Data from Multiple Static Loads and Vibration Tests," Dynamics Specialists Conference, AIAA Paper 19941714, 1994.

[12] Jaishi, B., and Ren, W.-X., "Damage Detection by Finite Element Model Updating Using Modal Flexibility Residual," Journal of Sound and Vibration, Vol. 290, Nos. 1-2, 2006, pp. 369-387. doi:10.1016/j.jsv.2005.04.006

[13] Avitabile, P., "Twenty Years of Structural Dynamic Modification-A Review," Sound and Vibration, Vol. 37, No. 1, 2003, pp. 14-27.

[14] Chen, G., "FE Model Validation for Structural Dynamics," Ph.D. Thesis, Mechanical Engineering Dept., Univ. of London, London, 2001.

[15] Friswell, M. I., and Mottershead, J. E., Finite Element Model Updating in Structural Dynamics, Kluwer, Dordrecht, The Netherlands, 1995, pp. 226-227.

[16] Lung, S.-F., and Pak, C.-G., "Updating the Finite Element Model of the Aerostructures Test Wing Using Ground Vibration Test Data," NASA TM-2009-214646, 2009.

[17] Pak, C.-G., "Preliminary Development of an Object-Oriented Optimization Tool," NASA TM-2011-216419, 2011.

[18] "MSC/NASTRAN Quick Reference Guide Version 69," MSC Software, Newport Beach, CA, 1996.

[19] "DOT Design Optimization Tools User's Manual Version 5.0," Vanderplaats Research and Development, Colorado Springs, CO, 2001.
[20] Charbonneau, P., and Knapp, B., "A User's Guide to PIKAIA 1.0," National Center for Atmospheric Research, Boulder, CO, 1995, p. 2.

[21] Erol, O. K., and Eksin, I., "A New Optimization Method: Big Bang-Big Crunch," Advances in Engineering Software, Vol. 37, No. 2, 2006, pp. 106-111. doi:10.1016/j.advengsoft.2005.04.005

[22] Camp, C. V., "Design of Space Trusses Using Big Bang-Big Crunch Optimization," Journal of Structural Engineering, Vol. 133, No. 7, 2007, pp. 999-1008.

doi:10.1061/(ASCE)0733-9445(2007)133:7(999)

[23] Kaveh, A., and Talatahari, S., "Size Optimization of Space Trusses Using Big Bang-Big Crunch Algorithm," Computers and Structures, Vol. 87, Nos. 17-18, 2009, pp. 1129-1140. doi:10.1016/j.compstruc.2009.04.011

[24] Tang, H., Zhou, J., Xue, S., and Xie, L., "Big Bang-Big Crunch Optimization for Parameter Estimation in Structural Systems," Mechanical Systems and Signal Processing, Vol. 24, No. 8, 2010, pp. 2888-2897. doi:10.1016/j.ymssp.2010.03.012

[25] Pak, C.-G., and Li, W., "Multidisciplinary Design, Analysis, and Optimization Tool Development Using a Genetic Algorithm," Proceedings of the 26th International Congress of the Aeronautical Sciences, Anchorage, AK, 2008.

[26] O'Callahan, J., Avitabile, P., and Riemer, R., "System Equivalent Reduction Expansion Process," Proceedings of the 7th International Modal Analysis Conference, Society for Experimental, NV, June 1989, pp. 29-37.

[27] "Test Requirements for Launch, Upper-Stage, and Space Vehicles," MIL-STD-1540C, Sec. 6.2.10, Sept. 1994.

[28] NASATechnical Standard, "Load Analysis of Spacecraft and Payloads," NASA STD-5002, Sec. 4.2.6.d, June 1996.

[29] "ZAERO User's Manual Version 8.2," ZONA Technology, Inc., Scottsdale, AZ, 2008 